\title{
INFINITE SYSTEM OF BROWNIAN BALLS WITH INTERACTION: THE NON-REVERSIBLE CASE*
}

\author{
Myriam Fradon ${ }^{1}$ AND Sylvie Relly ${ }^{2,3}$
}

\begin{abstract}
We consider an infinite system of hard balls in $\mathbb{R}^{d}$ undergoing Brownian motions and submitted to a smooth pair potential. It is modelized by an infinite-dimensional stochastic differential equation with an infinite-dimensional local time term. Existence and uniqueness of a strong solution is proven for such an equation with fixed deterministic initial condition. We also show that Gibbs measures are reversible measures.
\end{abstract}

Mathematics Subject Classification. 60H10,60K35.

Invited paper accepted September 2005.

\section{INTRODUCTION}

The aim of this paper is to construct and analyze an infinite system of interacting hard balls undergoing Brownian motions in $\mathbb{R}^{d}$ and starting from a fixed initial condition.

R. Lang [5] constructed in a pioneer paper the reversible solution of an infinite gradient system of Brownian particles (i.e. balls with radius 0 , that is reduced to points) submitted to a smooth pair interaction. It is a so-called equilibrium dynamics in statistical physics, since this process has a time-stationary distribution. J. Fritz solved some years later in [3] the non-reversible case, which occurs when the initial distribution is no more Gibbsian. For this type of systems, the main difficulty comes from a possible explosion (i.e. an infinite number of particles can enter in a finite volume after a finite time).

On another side, a reversible system of infinitely many Brownian hard balls (without external potential) was studied by H. Tanemura [8]. He constructs a unique solution to an infinite-dimensional Skohorod type equation where the hard core situation - balls can not overlap - appears as a local time term in addition to the basic Brownian motion. The (reversible) initial condition is ditributed like a Gibbs measure associated to the hard core potential.

In the present paper, the model is a mixture of both Lang's and Tanemura's models. We deal with Brownian motions submitted to the sum of a hard core potential and a smooth finite range pair potential. In [2] we proved

\footnotetext{
Keywords and phrases. Stochastic differential equation, local time, hard core potential, Gibbs measure, reversible measure.

* Ces quelques pages sont dédiées avec reconnaissance et amitié à Nicole, qui nous fit découvrir les beautés de beaucoup de planètes mathématiques, en particulier celle des diffusions infini-dimensionnelles.

1 Laboratoire CNRS 8524, UFR de Mathématiques, Université des Sciences et Technologies de Lille, 59655 Villeneuve d'Ascq Cedex, France; Myriam.Fradon@univ-lille1.fr

2 Institut für Mathematik, Universität Potsdam, Am Neuen Palais, 14415 Potsdam, Germany; roelly@math.uni-potsdam.de

3 On leave of absence Centre de Mathématiques Appliquées, UMR CNRS 7641, École Polytechnique, 91128 Palaiseau Cédex, France.
}

(c) EDP Sciences, SMAI 2007 
existence and uniqueness of a reversible solution of equation $(\mathcal{E})$, under the condition that the initial distribution is Gibbs with a small mean density of spheres. We propose here the construction of a strong non-reversible solution of $(\mathcal{E})$, in the sense that the initial condition can be any deterministic configuration in a set of allowed configurations which is clearly identified.

Although some techniques in the proof of the main results are similar to those in [2], we adopt a new pathwise approach for the construction of the solution of $(\mathcal{E})$ which is much finer than in [2], where the time-stationarity of the solution was used at several places. Moreover we make explicit in Theorem 1.3 the set of allowed initial configurations, and prove that any Gibbs measure associated with the dynamical interaction carries a.s. this set.

In Section 1 we present the infinite dimensional equation $(\mathcal{E})$ and we state the results. The sequence of approximating solutions is built in Section 2.1. Furthermore, we prove in Section 2 technical estimates needed in Section 3, for the convergence of the approximations. Finally, Section 4 is devoted to complete the proof of the main results.

\section{Dynamics AND MAIN Results}

\subsection{Configuration spaces}

The particles we deal with in the present paper move in $\mathbb{R}^{d}$, for a fixed $d \geqslant 2$, endowed with the Euclidian norm denoted by ||$. B(y, \rho)$ will denote the closed ball centered in $y \in \mathbb{R}^{d}$ with radius $\rho \geq 0$ and more generally, for any $A \subset \mathbb{R}^{d}$, we define

$$
B(A, \rho)=\left\{y \in \mathbb{R}^{d} \text { such that } d(y, A) \leqslant \rho\right\}
$$

where $d(y, A)$ denotes the Euclidian distance between $y$ and $A$. The volume of a subset $A$ in $\mathbb{R}^{d}$ is also denoted by $|A|$.

The modelization of point configurations may be done in two equivalent ways. The first possibility is to represent an $n$ points configuration in $\mathbb{R}^{d}$ as a subset (with multiplicity) of cardinal $n$ in $\mathbb{R}^{d}$, that is as an equivalence class on $\left(\mathbb{R}^{d}\right)^{n}$ under the action of the permutation group $\Sigma_{n}$ on $\{1, \ldots, n\}$. The second possibility is to modelize it as a point measure $\sum_{i=1}^{n} \delta_{\xi_{i}}$ on $\mathbb{R}^{d}$. More generally, the set of all point configurations in $\mathbb{R}^{d}$ will be the set $\mathcal{M}$ of all point Radon measures on $\mathbb{R}^{d}$ :

$$
\mathcal{M}=\left\{\xi=\sum_{i \in I} \delta_{\xi_{i}} \text { such that } I \subset \mathbb{N}, \xi_{i} \in \mathbb{R}^{d} \text { and for all } \Lambda \text { compact in } \mathbb{R}^{d}, \xi(\Lambda)<+\infty\right\} .
$$

$\mathcal{M}$ is endowed with the topology of vague convergence. By simplicity, we will identify any point measure $\xi \in \mathcal{M}$ with the subset of $\mathbb{R}^{d}\left\{\xi_{i}, i \in I\right\}$ corresponding to its support and with the representants of this subset in $\left(\mathbb{R}^{d}\right)^{I}$, writing for example $\xi_{\Lambda}=\xi \cap \Lambda$ for the restriction of this configuration to $\Lambda \subset \mathbb{R}^{d}, \xi \eta$ for the concatenation of both configurations $\xi$ and $\eta$. $\mathcal{M} \cap\left(\mathbb{R}^{d}\right)^{n}$ is the set of all $n$ points configurations.

A function $g$ on $\mathcal{M}$ is called $\mathcal{C}^{1}$ if for each $\gamma \in \mathcal{M}, y \mapsto g(y \gamma)=g\left(\delta_{y}+\gamma\right)$ is a $\mathcal{C}^{1}$-function on $\mathbb{R}^{d}$. $D_{x} g(x \gamma)$ denotes its derivative at $y=x$.

We introduce the following notations.

- For $\Lambda \subset \mathbb{R}^{d}, N_{\Lambda}$ is the counting variable on $\mathcal{M}: N_{\Lambda}(\xi)=\operatorname{Card}\left\{i \in \mathbb{N}, \xi_{i} \in \Lambda\right\}$.

- For $\Lambda \subset \mathbb{R}^{d}, \mathcal{B}_{\Lambda}$ is the $\sigma$-algebra on $\mathcal{M}$ generated by the sets $\left\{N_{A}=n\right\}, n \in \mathbb{N}, A \subset \Lambda, A$ bounded.

- $\pi\left(\right.$ resp. $\left.\pi_{\Lambda}\right)$ is the Poisson process on $\mathbb{R}^{d}$ (resp. on $\Lambda$ ) with intensity measure the Lebesgue measure $\mathrm{d} y\left(\right.$ resp. $\left.\left.\mathrm{d} y\right|_{\Lambda}\right)$.

- For $z>0, \pi^{z}$ (resp. $\pi_{\Lambda}^{z}$ ) is the Poisson process on $\mathbb{R}^{d}$ (resp. on $\Lambda$ ) with activity $z$, that is with intensity measure $z \mathrm{~d} y$ (resp. $\left.z \mathrm{~d} y\right|_{\Lambda}$ ).

The particles we deal with in this paper are not reduced to points but are hard balls or spheres of diameter $r$, for a fixed $r>0$. So the set of allowed configurations is the following subset of $\mathcal{M}$ :

$$
\mathcal{A}=\left\{\xi \in \mathcal{M} \text { such that } \forall i \neq j\left|\xi_{i}-\xi_{j}\right| \geqslant r\right\} .
$$


Remark 1.1. We study here the evolution of a particles configuration under the influence of an interaction potential with finite range $R$. Then a fixed particle can interact with at most a finite number $\bar{N}$ of particles. $\bar{N}$ only depends on $d$ and $R / r$ and is clearly bounded by $\frac{(R+r / 2)^{d}}{(r / 2)^{d}}=(1+2 R / r)^{d}$.

\subsection{Interaction potential and associated Gibbs measures}

For a complete description in a general framework of the concepts introduced in this subsection, we refer the reader to $[4]$.

We are dealing with hard balls with diameter $r$ submitted to the action of a pair potential, which is a function on $\mathbb{R}^{d}$ of class $\mathrm{C}^{2}$ with finite range $R>r$, i.e. satisfying $\varphi(x)=0$ if $|x| \geqslant R$ and $\varphi(x)=\varphi(-x)$. Due to the hard core situation the values of $\varphi(x)$ may be chosen arbitrarily for $|x|<r$. In particular, one can assume without restriction that $\varphi$ vanishes in a neighborhood from 0 and that $\nabla \varphi(0)=0$. Since $\varphi$ has compact support, it is bounded from below: the smallest value of interaction between two particles is given by

$$
\underline{\varphi}=\inf _{|x| \geqslant r} \varphi(x) \leqslant 0
$$

If this real constant is zero there exists only repulsion between the balls; if it is negative there exists an attraction domain around each ball.

The energy of a configuration $\xi \in \mathcal{M}$ submitted to the potential $\varphi$ in the compact volume $\Lambda \subset \mathbb{R}^{d}$ with the boundary condition $\eta \in \mathcal{M}$ is given by:

$$
E_{\Lambda}(\xi \mid \eta)= \begin{cases}\frac{1}{2} \sum_{\xi_{i}, \xi_{j} \in \Lambda} \varphi\left(\xi_{i}-\xi_{j}\right)+\sum_{\xi_{i} \in \Lambda, \eta_{j} \in \Lambda^{c}} \varphi\left(\xi_{i}-\eta_{j}\right) & \text { if } \xi_{\Lambda} \eta_{\Lambda^{c}} \in \mathcal{A} \\ +\infty & \text { otherwise }\end{cases}
$$

(the condition $\xi_{\Lambda} \eta_{\Lambda^{c}} \in \mathcal{A}$ corresponds to configurations for which $\xi_{\Lambda} \in \mathcal{A}, \eta_{\Lambda^{c}} \in \mathcal{A}$ and no ball of $\eta_{\Lambda^{c}}$ is overlapping a ball of $\left.\xi_{\Lambda}\right)$. The energy is well defined since both sums contain no more than $\frac{|B(\Lambda, r / 2)|}{|B(0, r / 2)|} \bar{N}$ terms, see Remark 1.1. Moreover, $\mathrm{e}^{-E_{\Lambda}(\xi \mid \eta)}$ vanishes as soon as the configuration $\xi_{\Lambda} \eta_{\Lambda^{c}}$ is not allowed.

We now define the set $\mathcal{G}(z)$ of Gibbs measures on $\mathcal{A}$ associated to the potential $\varphi$ with activity parameter $z \in \mathbb{R}^{+}$. For each compact subset $\Lambda$ of $\mathbb{R}^{d}$, let us define a local density function with respect to the Poisson process $\pi_{\Lambda}^{z}$ by:

$$
f_{\Lambda}^{z}(\xi \mid \eta)=\frac{1}{Z_{z}^{\Lambda, \eta}} \exp \left(-E_{\Lambda}(\xi \mid \eta)\right)
$$

where the so-called partition function $Z_{z}^{\Lambda, \eta}$ is the renormalizing constant:

$$
Z_{z}^{\Lambda, \eta}=\mathrm{e}^{-z|\Lambda|}\left(1+\sum_{n=1}^{+\infty} \frac{z^{n}}{n !} \int_{\Lambda^{n}} \exp -E_{\Lambda}\left(y_{1} \cdots y_{n} \mid \eta\right) \mathrm{d} y_{1} \cdots \mathrm{d} y_{n}\right)
$$

Due to the hard core, the above series is only a finite sum and $0<Z_{z}^{\Lambda, \eta}<+\infty$.

Definition 1.2. A probability measure $\mu$ on $\mathcal{M}$ belongs to the set $\mathcal{G}(z)$ of Gibbs measures on hard balls with activity $z$ and associated potential $\varphi$ if and only if, for each compact subset $\Lambda \subset \mathbb{R}^{d}$,

$$
\mathrm{d} \mu\left(\xi \mid \mathcal{B}_{\Lambda^{c}}\right)(\eta)=f_{\Lambda}^{z}(\xi \mid \eta) \mathrm{d} \pi_{\Lambda}^{z}(\xi) \quad \text { for } \mu-\text { a.e. } \eta \text {. }
$$

Remark that any Gibbs measure in $\mathcal{G}(z)$ has its support included in $\mathcal{A}$. Dobrushin proved in [1], using compactness argument, that there exists at least one element in $\mathcal{G}(z)$ when the potential contains a hard core component. Furthermore the set $\mathcal{G}(z)$ is convex and compact. About the cardinality of $\mathcal{G}(z)$, remarking that the sum of 
the hard core and the smooth potential $\varphi$ is superstable and lower regular in the sense of Ruelle [6], we do the following remarks:

- If $z$ is small enough Ruelle proved that uniqueness holds. In our case, a sufficient condition would be: $z \leq \mathrm{e}^{\bar{N} \underline{\varphi}-1}\left(|B(0, r)|+\int \mathbb{I}_{r<|y|<R}\left|1-\mathrm{e}^{-\varphi(y)}\right| \mathrm{d} y\right)^{-1}$.

- For $z$ large enough it is conjectured (see [4]) - but still not proved - that phase transition occurs: Card $\mathcal{G}(z)>1$.

\subsection{The stochastic equation $(\mathcal{E})$ and statement of the main results}

Let $(\Omega, \mathcal{F}, P)$ be a probability space with a right continuous filtration $\left\{\mathcal{F}_{t}\right\}_{t \geqslant 0}$ such that each $\mathcal{F}_{t}$ contains all $P$ - negligible sets and let $\left(W_{i}(t), t \geqslant 0\right)_{i \in \mathbb{N}}$ be a family of $\mathcal{F}_{t}$-adapted independent $d$-dimensional Brownian motions.

Let us denote $\mathcal{C}\left(\mathbb{R}^{+}, \mathcal{M}\right)$ (respectively $\left.\mathcal{C}\left(\mathbb{R}^{+}, \mathcal{A}\right)\right)$ the set of continuous $\mathcal{M}$-valued (resp. $\mathcal{A}$-valued) paths on $\mathbb{R}^{+}$, endowed with the topology of uniform convergence on each compact time interval.

Let $\varphi$ be the smooth pair potential with finite range $R$ introduced in the previous subsection. We consider the following infinite gradient system of stochastic equations satisfied by the Brownian balls:

$$
(\mathcal{E})\left\{\begin{array}{l}
\text { For } i \in \mathbb{N}, t \in \mathbb{R}^{+}, \\
X_{i}(t)=X_{i}(0)+W_{i}(t)-\frac{1}{2} \sum_{j \in \mathbb{N}} \int_{0}^{t} \nabla \varphi\left(X_{i}(s)-X_{j}(s)\right) \mathrm{d} s+\sum_{j \in \mathbb{N}} \int_{0}^{t}\left(X_{i}(s)-X_{j}(s)\right) \mathrm{d} L_{i j}(s)
\end{array}\right.
$$

where

- $\left(X_{i}(t), t \geqslant 0\right)_{i \in \mathbb{N}} \in \mathcal{C}\left(\mathbb{R}^{+}, \mathcal{A}\right)$ satisfies $\left|X_{i}(t)-X_{j}(t)\right| \geqslant r$ for $t \geqslant 0$ and $i \neq j$;

- $\left(L_{i j}(t), t \geqslant 0\right)_{i, j \in \mathbb{N}}$ is a family of non-decreasing $\mathbb{R}^{+}$-valued continuous processes satisfying:

$$
L_{i j}(0)=0, \quad L_{i j} \equiv L_{j i} \quad \text { and } \quad L_{i j}(t)=\int_{0}^{t} \mathbb{1}_{\left|X_{i}(s)-X_{j}(s)\right|=r} \mathrm{~d} L_{i j}(s), \quad L_{i i} \equiv 0 .
$$

A solution of the system $(\mathcal{E})$ with initial condition $x \in \mathcal{A}$ is a family $\left(X_{i}^{x}(t), L_{i j}^{x}(t), t \geqslant 0, i, j \in \mathbb{N}\right)$ of processes such that equation $(\mathcal{E})$ is satisfied with $X(0)=x$.

The main results of this paper are the following theorems.

Theorem 1.3. The stochastic equation $(\mathcal{E})$ admits a solution with values in $\mathcal{A}$ for any deterministic initial configuration which belongs to the set $\underline{\underline{\mathcal{A}}} \subset \mathcal{A}$ defined by $\underline{\underline{\mathcal{A}}}=\left\{x \in \mathcal{A}: P\left(\Omega_{0}^{x} \cap \Omega_{1}^{x}\right)=1\right\}$ (sets $\Omega_{0}^{x}$ and $\Omega_{1}^{x}$ are given in (15) and (23)). This solution is unique as element of $\mathcal{C} \subset \mathcal{C}\left(\mathbb{R}^{+}, \mathcal{A}\right)$, a subset of paths with some regularity defined in Proposition 4.4 .

Theorem 1.4. If the initial configuration of the stochastic equation $(\mathcal{E})$ is random with distribution $\mu \in \mathcal{G}(z)$ for some $z>0$ and $\mu(\underline{\underline{\mathcal{A}}})=1$, then this solution is time-reversible, that is its law is invariant with respect to the time reversal.

Proposition 1.5. Let $z_{c}$ be a critical value of the activity given by: $z_{c}=\frac{\exp (2 \bar{N} \underline{\varphi})}{\left(R^{d}-r^{d}\right) \mid \overline{B(0,1) \mid}}$. Any Gibbs measure $\mu \in \mathcal{G}(z)$ with $0<z<z_{c}$ has its support included in $\underline{\underline{\mathcal{A}}}$.

Remark 1.6. The existence of a critical value for the activity $z$ is related to the still open problem of percolation for the hard core continuous system. The critical value $z_{c}$ given here appears for technical reasons in Corollary 2.5, where a percolation type estimate is computed. 


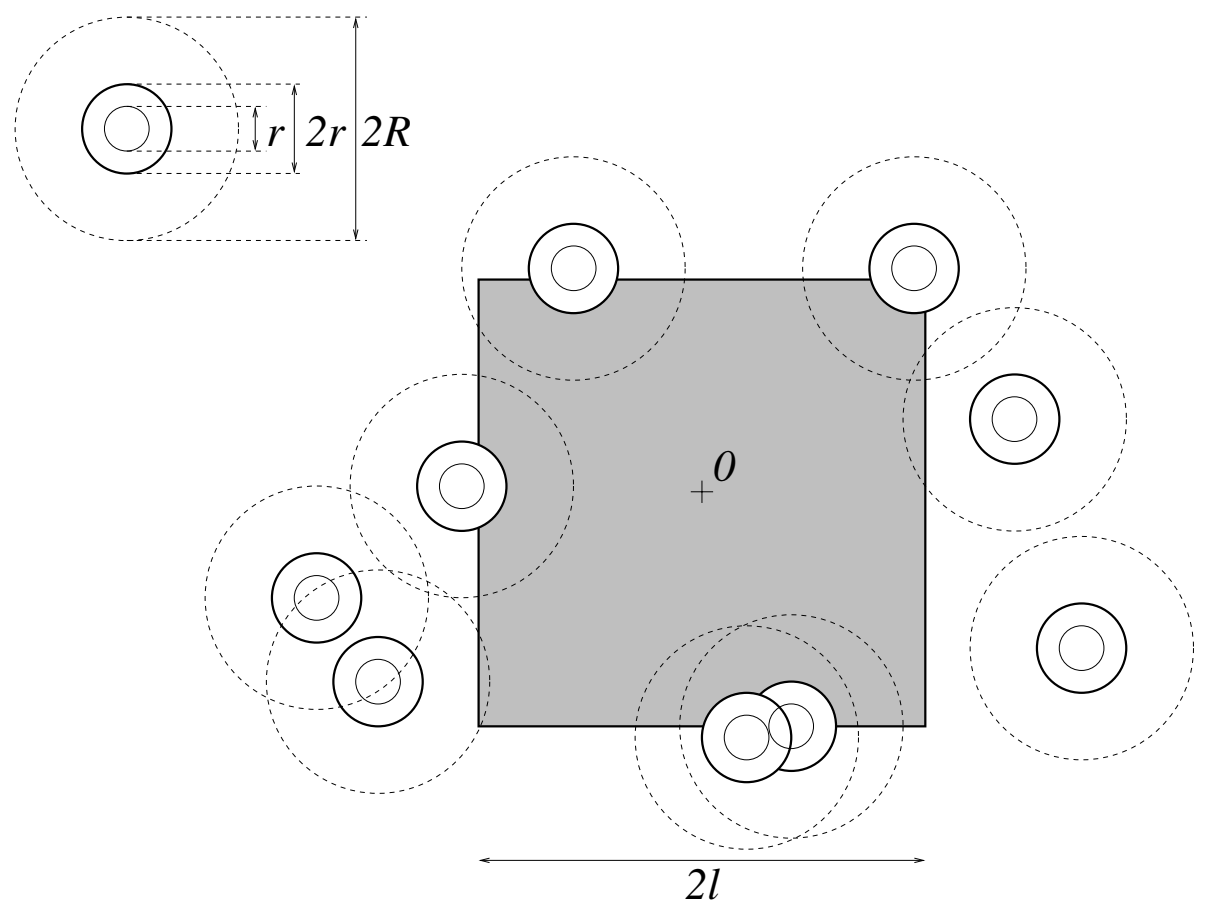

Figure 1. Particles of $\eta_{\Lambda_{l}}$ are represented; the grey area is the domain where $\psi^{l, \eta}$ vanishes.

\section{Approximating processes and estimates on the paths set}

In this whole subsection, $l \in \mathbb{N}^{*}$ is fixed. To simplify we restrict the study of the paths on the time interval $[0,1]$. It is obvious that all the results in the sequel hold true on any time interval $[0, T], T \geqslant 1$, up to a change of constants.

\subsection{Construction of approximating processes}

We construct the approximating process $X^{l, x}$ in order that it "essentially" stays in the bounded cube $\Lambda_{l}=$ $[-l, l]^{d}$ (in a sense which will be clear soon). To obtain such a behavior, we introduce in the equation $(\mathcal{E})$ a supplementary gradient drift $\nabla \psi^{l, \eta}$ which vanishes in a subset of $\Lambda_{l}$ and is repulsive outside of $\Lambda_{l}$.

More precisely, for any allowed configuration $\eta \in \mathcal{A}$ which support is disjoint to $\Lambda_{l}$, we fix a $\mathbb{R}^{+}$-valued function $\psi^{l, \eta}$ on $\mathbb{R}^{d}$ which is $\mathrm{C}^{2}$ with bounded derivatives and vanishes on each (and only on) $y \in \Lambda_{l}$ such that $y \eta$ is an allowed configuration (see Fig. 1), that is

$$
\psi^{l, \eta}(y)=0 \quad \Leftrightarrow \quad y \in \Lambda_{l}=[-l, l]^{d} \text { and } y \eta \in \mathcal{A} \quad \Leftrightarrow \quad y \in \Lambda_{l}=[-l, l]^{d} \text { and } d(y, \eta) \geqslant r .
$$

We extend the definition of $\psi^{l, \eta}$ to any configuration $\eta \in \mathcal{A}$ by: $\psi^{l, \eta}=\psi^{l, \eta_{\Lambda} c}$. We also choose the family $\left(\psi^{l, \eta}\right)_{l}$ such that, for every $\eta \in \mathcal{A}$,

$$
\sum_{l \in \mathbb{N}^{*}} \int_{\mathbb{R}^{d}} \mathbb{I}_{\psi^{l, \eta}(y)>0} \exp \left(-\psi^{l, \eta}(y)\right) \mathrm{d} y \leqslant 1
$$


For $\eta \in \mathcal{A}$ and $n \in \mathbb{N}^{*}$, let us now define the $n$-dimensional stochastic differential equation:

$$
\left(\mathcal{E}_{n}^{l, \eta}\right)\left\{\begin{aligned}
\forall i \in\{1, \ldots, n\}, & \text { for } 0 \leq t \leq 1, \\
\mathrm{~d} X_{i}(t)=\mathrm{d} W_{i}(t) & -\frac{1}{2}\left(\sum_{j=1, \ldots, n} \nabla \varphi\left(X_{i}(t)-X_{j}(t)\right)+\sum_{j: \eta_{j} \in \Lambda^{c}} \nabla \varphi\left(X_{i}(t)-\eta_{j}\right)\right) \mathrm{d} t \\
& -\frac{1}{2} \nabla \psi^{l, \eta}\left(X_{i}(t)\right) \mathrm{d} t+\sum_{j=1, \ldots, n}\left(X_{i}(t)-X_{j}(t)\right) \mathrm{d} L_{i j}(t)
\end{aligned}\right.
$$

with $L_{i j} \equiv L_{j i}$ for all $i$ and $j$ and $L_{i j}(t)=\int_{0}^{t} \mathbb{1}_{\left|X_{i}(s)-X_{j}(s)\right|=r} \mathrm{~d} L_{i j}(s) .\left(\mathcal{E}_{n}^{l, \eta}\right)$ is a $n$-dimensional stochastic differential equation reflected in $\mathcal{A} \cap\left(\mathbb{R}^{d}\right)^{n}$ with gradient drift $-\frac{1}{2} \nabla \beta_{n}^{l, \eta}$ where

$$
\beta_{n}^{l, \eta}\left(x_{1}, \ldots, x_{n}\right)=\sum_{i=1, \ldots, n}\left(\psi^{l, \eta}\left(x_{i}\right)+\frac{1}{2} \sum_{\substack{j=1, \ldots, n \\ j \neq i}} \varphi\left(x_{i}-x_{j}\right)+\sum_{j: \eta_{j} \in \Lambda^{c}} \varphi\left(x_{i}-\eta_{j}\right)\right) .
$$

Since the drift $-\frac{1}{2} \nabla \beta_{n}^{l, \eta}$ is bounded and Lipschitz continuous, $\left(\mathcal{E}_{n}^{l, \eta}\right)$ admits a unique strong solution for each initial $n$-point configuration $x \in \mathcal{A} \cap\left(\mathbb{R}^{d}\right)^{n}$ (see Th. 5.1 of [7]). We denote this solution by $X^{l, \eta, n}(x, \cdot)$. For a general initial configuration $x \in \mathcal{A}$, we extend the above process as follows:

$$
X^{l, x}(\cdot)=X^{l, \eta, n}\left(x_{\Lambda_{l}}, \cdot\right) x_{\Lambda_{l}^{c}}
$$

where $\eta=x_{\Lambda_{l}^{c}}$ and $n=\operatorname{Card}\left(x \cap \Lambda_{l}\right)$. It is an $\mathcal{M}$-valued (not necessarily $\mathcal{A}$-valued) process with initial configuration $x$. Particles which are initially in $\Lambda_{l}$ move like the $\left(\mathcal{E}_{n}^{l, \eta}\right)$-dynamics and the other ones stay fixed outside $\Lambda_{l}$.

The solution of $\left(\mathcal{E}_{n}^{l, \eta}\right)$ with initial distribution $\nu_{n}^{l, \eta}$ is reversible, where $\nu_{n}^{l, \eta}$ is the finite measure defined on $\left(\mathbb{R}^{d}\right)^{n}$ by

$$
\mathrm{d} \nu_{n}^{l, \eta}\left(x_{1}, \ldots, x_{n}\right)=\exp \left(-\beta_{n}^{l, \eta}\left(x_{1}, \ldots, x_{n}\right)\right) \mathbb{I}_{\mathcal{A}}\left(x_{1}, \ldots, x_{n}\right) \mathrm{d} x_{1} \ldots \mathrm{d} x_{n} .
$$

$Q_{n}^{l, \eta}$ denotes the time-reversible law of $X^{l, \eta, n}$ starting from $\nu_{n}^{l, \eta}$ :

$$
Q_{n}^{l, \eta}=\int P\left(X^{l, \eta, n}(x, \cdot) \in .\right) \mathrm{d} \nu_{n}^{l, \eta}(x) .
$$

The probability measure $\mu_{z}^{l, \eta}$ on $\bigcup_{n=0}^{+\infty}\left(\mathbb{R}^{d}\right)^{n}$ is defined for $A_{0} \times A_{1} \times \cdots \times A_{n} \times \cdots$ by:

$$
\mu_{z}^{l, \eta}\left(A_{0} \times A_{1} \times \cdots \times A_{n} \times \cdots\right)=\frac{\mathrm{e}^{-z 2^{d} l^{d}}}{Z_{z}^{l, \eta}} \sum_{n=0}^{+\infty} \frac{z^{n}}{n !} \nu_{n}^{l, \eta}\left(A_{n}\right)
$$

where $Z_{z}^{l, \eta}=\sum_{n=0}^{+\infty} \frac{z^{n}}{n !} \nu_{n}^{l, \eta}\left(\left(\mathbb{R}^{d}\right)^{n}\right)$ (with the convention $\nu_{0}^{l, \eta}(\{\emptyset\})=1$ ).

Similarly, consider the probability measure on $\bigcup_{n=0}^{+\infty} \mathcal{C}\left([0,1],\left(\mathbb{R}^{d}\right)^{n}\right)$ defined by

$$
Q_{z}^{l, \eta}=\frac{\mathrm{e}^{-z 2^{d} l^{d}}}{Z_{z}^{l, \eta}} \sum_{n=0}^{+\infty} \frac{z^{n}}{n !} Q_{n}^{l, \eta}
$$


This probability measure is time reversal invariant and has a support in $\mathcal{A}$, as a mixing of $\mathcal{A}$-supported measures.

We now want to prove that the probability of trajectories which interact too much, vanishes asymptotically when $l \rightarrow+\infty$. We will use this result to construct the limit of $\left(X^{l, x}\right)_{l}$ in the Section 3 .

Regular paths are $\omega$ 's such that each particle interacts only with a finite number of particles during a finite time interval ; $X^{l, x}(\omega)$ is then the (unique) solution of a finite dimensional equation. Bad $\omega$ 's are paths such that at least one particle interacts with a great number of other ones, either because it moves very fast (see Sect. 2.2), or because it belongs to a large chain of particles where each one interacts with its neighbors (see Sect. 2.3).

\subsection{Paths with high velocity}

We obtain here an estimate of the probability that a particle moves "too fast". In order to establish such an estimate, done in Proposition 2.2, we first compute the probability of fast motion between two fixed bounded domains in $\mathbb{R}^{d}$.

For every bounded subsets $A_{0}$ and $A_{1}$ of $\mathbb{R}^{d}$ and every $\varepsilon>0$ and $\left.\left.\delta \in\right] 0,1\right]$, let $\mathcal{F} m\left(A_{0}, A_{1}, \varepsilon, \delta\right)$ denote the event "at least a particle goes from $A_{0}$ to $A_{1}$ with an oscillation greater than $\varepsilon$ in a time interval smaller than $\delta "$, i.e.

$$
\mathcal{F} m\left(A_{0}, A_{1}, \varepsilon, \delta\right)=\left\{X \in \mathcal{C}([0,1], \mathcal{A}), \quad \exists i \text { s.t. } X_{i}(0) \in A_{0}, X_{i}(1) \in A_{1} \text { and } w\left(X_{i}, \delta\right)>\varepsilon\right\}
$$

where $w\left(X_{i}, \delta\right)=\sup _{\substack{|t-s|<\delta \\ 0 \leqslant s, t \leqslant 1}}\left|X_{i}(t)-X_{i}(s)\right|$ is the usual modulus of continuity of the path $X_{i}$ on $[0,1]$.

Lemma 2.1. For each $A_{0}, A_{1} \subset \mathbb{R}^{d}$ bounded, each $\left.\left.\varepsilon>0, \delta \in\right] 0,1\right], \eta \in \mathcal{A}$ and $n \in \mathbb{N}^{*}$, we have:

$$
\begin{aligned}
\left.Q_{n}^{l, \eta}\left(\mathcal{F} m\left(A_{0}, A_{1}, \varepsilon, \delta\right)\right)\right) & =\int P\left(X^{l, \eta, n}(x, \cdot) \in \mathcal{F} m\left(A_{0}, A_{1}, \varepsilon, \delta\right)\right) \mathrm{d} \nu_{n}^{l, \eta}(x) \\
& \leqslant 41 n \mathrm{e}^{-2 \bar{N} \underline{\varphi}} \nu_{n-1}^{l, \eta}\left(\left(\mathbb{R}^{d}\right)^{n-1}\right) \frac{1}{\delta} \exp \left(-\frac{\varepsilon^{2}}{5 \delta}\right) \int_{\mathbb{R}^{d}}\left(\mathbb{I}_{A_{0}}+\mathbb{1}_{A_{1}}\right) \mathrm{e}^{-\psi^{l, \eta}} \mathrm{d} y
\end{aligned}
$$

and

$$
\left.Q_{z}^{l, \eta}\left(\mathcal{F} m\left(A_{0}, A_{1}, \varepsilon, \delta\right)\right)\right) \leqslant\left(41 z \mathrm{e}^{-2 \bar{N} \underline{\varphi}}\right) \frac{1}{\delta} \exp \left(-\frac{\varepsilon^{2}}{5 \delta}\right) \int_{\mathbb{R}^{d}}\left(\mathbb{I}_{A_{0}}+\mathbb{1}_{A_{1}}\right) \mathrm{e}^{-\psi^{l, \eta}} \mathrm{d} y .
$$

From this lemma proved below, we easily deduce an estimate of the probability, under $Q_{z}^{l, \eta}$, that a particle starting from $B(0, K)$ moves too fast. For every $K \in \mathbb{N}^{*}, \varepsilon>0$ and $\left.\left.\delta \in\right] 0,1\right]$, let $\mathcal{F} m(K, \varepsilon, \delta)$ be the following event:

$$
\mathcal{F} m(K, \varepsilon, \delta)=\left\{X \in \mathcal{C}([0,1], \mathcal{A}) \quad \text { such that } \quad \exists i, X_{i}(0) \in B(0, K) \text { and } w\left(X_{i}, \delta\right)>\varepsilon\right\} .
$$

Proposition 2.2. The following upper bounds hold:

$$
\begin{aligned}
\left.\left.\forall K \in \mathbb{N}^{*} \quad \forall \varepsilon>0 \quad \forall \delta \in\right] 0,1\right] \quad \forall \eta \in \mathcal{A} \\
Q_{n}^{l, \eta}(\mathcal{F} m(K, \varepsilon, \delta)) \leqslant n C_{d} \mathrm{e}^{-2 \bar{N} \underline{\varphi}} \nu_{n-1}^{l, \eta}\left(\left(\mathbb{R}^{d}\right)^{n-1}\right) \frac{1}{\delta} \exp \left(-\frac{\varepsilon^{2}}{6 \delta}\right) K^{d} \\
Q_{z}^{l, \eta}(\mathcal{F} m(K, \varepsilon, \delta)) \leqslant z C_{d} \mathrm{e}^{-2 \bar{N} \underline{\varphi}} \frac{1}{\delta} \exp \left(-\frac{\varepsilon^{2}}{6 \delta}\right) K^{d}
\end{aligned}
$$

where $C_{d}$ is a constant depending only on dimension d. Similarly one has:

$$
\begin{aligned}
\left.\left.\forall K \in \mathbb{N}^{*} \quad \forall \varepsilon>0 \quad \forall \delta \in\right] 0,1\right] \quad \forall \eta \in \mathcal{A} \\
Q_{n}^{l, \eta}(\mathcal{F} m(K, \varepsilon, \delta)) \leqslant 246 n \mathrm{e}^{-2 \bar{N} \underline{\varphi}} \nu_{n-1}^{l, \eta}\left(\left(\mathbb{R}^{d}\right)^{n-1}\right) \frac{1}{\delta} \exp \left(-\frac{\varepsilon^{2}}{5 \delta}\right) \int_{\mathbb{R}^{d}} \mathrm{e}^{-\psi^{l, \eta}(y)} \mathrm{d} y \\
Q_{z}^{l, \eta}(\mathcal{F} m(K, \varepsilon, \delta)) \leqslant 246 z \mathrm{e}^{-2 \bar{N} \underline{\varphi}} \frac{1}{\delta} \exp \left(-\frac{\varepsilon^{2}}{5 \delta}\right) \int_{\mathbb{R}^{d}} \mathrm{e}^{-\psi^{l, \eta}(y)} \mathrm{d} y .
\end{aligned}
$$


Proof of Lemma 2.1. We first need an estimate of $Q_{n}^{l, \eta}\left(\mathcal{F} m\left(A_{0}, A_{1}, \varepsilon, \delta\right)\right)$.

Let $\left(X^{l, \eta, n}, L^{l, \eta, n}\right)$ denote the unique strong solution of $\left(\mathcal{E}_{n}^{l, \eta}\right)$ starting from $\nu_{n}^{l, \eta}$, and recall that the distribution $Q_{n}^{l, \eta}$ of $X^{l, \eta, n}$ is time reversible on $[0,1]$. By construction, the processes:

$$
\begin{aligned}
W_{i}(t)= & X_{i}^{l, \eta, n}(t)-X_{i}^{l, \eta, n}(0)+\frac{1}{2} \int_{0}^{t} \nabla_{i} \beta_{n}^{l, \eta}\left(X^{l, \eta, n}(s)\right) \mathrm{d} s \\
& -\int_{0}^{t} \sum_{j=1, \ldots, n}\left(X_{i}^{l, \eta, n}(s)-X_{j}^{l, \eta, n}(s)\right) \mathrm{d} L_{i j}^{l, \eta, n}(s), 1 \leqslant i \leqslant n, 0 \leqslant t \leqslant 1 \\
\text { and } \quad \widehat{W}_{i}(t)= & X_{i}^{l, \eta, n}(1-t)-X_{i}^{l, \eta, n}(1)+\frac{1}{2} \int_{1-t}^{1} \nabla_{i} \beta_{n}^{l, \eta}\left(X^{l, \eta, n}(s)\right) \mathrm{d} s \\
& -\int_{1-t}^{1} \sum_{j=1, \ldots, n}\left(X_{i}^{l, \eta, n}(s)-X_{j}^{l, \eta, n}(s)\right) \mathrm{d} L_{i j}^{l, \eta, n}(s), 1 \leqslant i \leqslant n, 0 \leqslant t \leqslant 1
\end{aligned}
$$

are both $n$-dimensional Brownian motions starting from 0. Remarking that

$$
\forall t \in[0,1] \quad X^{l, \eta, n}(t)-X^{l, \eta, n}(0)=\frac{1}{2}(W(t)+\widehat{W}(1-t)-\widehat{W}(1))
$$

and using the equality in law between $\left(X^{l, \eta, n}(1-\cdot), \widehat{W}\right)$ and $\left(X^{l, \eta, n}, W\right)$, we obtain:

$$
\begin{aligned}
Q_{n}^{l, \eta}\left(\mathcal{F} m\left(A_{0}, A_{1}, \varepsilon, \delta\right)\right)= & \int_{\left(\mathbb{R}^{d}\right)^{n}} P\left(\begin{array}{c}
\exists i \leqslant n \text { s.t. } X_{i}^{l, \eta, n}(x, 0) \in A_{0}, X_{i}^{l, \eta, n}(x, 1) \in A_{1} \text { and } \\
\sup _{\substack{|t-s|<\delta \\
0 \leqslant s, t \leqslant 1}}\left|W_{i}(t)-W_{i}(s)+\widehat{W}(1-t)-\widehat{W}(1-s)\right|>2 \varepsilon
\end{array}\right) \mathrm{d} \nu_{n}^{l, \eta}(x) \\
\leqslant & \int P\left(\exists i \leqslant n \text { s.t. } x_{i} \in A_{0} \text { and } w\left(W_{i}, \delta\right)>\varepsilon\right) \mathrm{d} \nu_{n}^{l, \eta}(x) \\
& +\int P\left(\exists i \leqslant n \text { s.t. } x_{i} \in A_{1} \text { and } w\left(W_{i}, \delta\right)>\varepsilon\right) \nu_{n}^{l, \eta}(x)
\end{aligned}
$$

The right hand side is smaller than

$$
\begin{aligned}
& \sum_{i=1}^{n} \nu_{n}^{l, \eta}\left(x_{i} \in A_{0}\right) P\left(w\left(W_{i}, \delta\right)>\varepsilon\right)+\sum_{i=1}^{n} \nu_{n}^{l, \eta}\left(x_{i} \in A_{1}\right) P\left(w\left(W_{i}, \delta\right)>\varepsilon\right) \\
& \leqslant n P\left(w\left(W_{1}, \delta\right)>\varepsilon\right)\left(\nu_{n}^{l, \eta}\left(x_{1} \in A_{0}\right)+\nu_{n}^{l, \eta}\left(x_{1} \in A_{1}\right)\right) .
\end{aligned}
$$

We know from Appendix 5 that

$$
P\left(w\left(W_{1}, \delta\right)>\varepsilon\right) \leqslant \frac{41}{\delta} \exp \left(-\frac{\varepsilon^{2}}{5 \delta}\right) .
$$

According to the definition (4) of $\beta_{n}^{l, \eta}$, since a particle interacts with at most $\bar{N}$ other particles (cf. Rem. 1.1):

$$
\beta_{n}^{l, \eta}\left(x_{1}, \ldots, x_{n}\right) \geqslant \psi^{l, \eta}\left(x_{1}\right)+2 \bar{N} \underline{\varphi}+\beta_{n-1}^{l, \eta}\left(x_{2}, \ldots, x_{n}\right)
$$

which implies that

$$
\begin{aligned}
\nu_{n}^{l, \eta}\left(x_{1} \in A_{0}\right) & =\int_{\left(\mathbb{R}^{d}\right)^{n}} \mathbb{I}_{x_{1} \in A_{0}} \mathbb{I}_{\mathcal{A}}\left(x_{1}, \ldots, x_{n}\right) \mathrm{e}^{-\beta_{n}^{l, \eta}\left(x_{1}, \ldots, x_{n}\right)} \mathrm{d} x_{1} \cdots \mathrm{d} x_{n} \\
& \leqslant \mathrm{e}^{-2 \bar{N} \underline{\varphi}} \nu_{n-1}^{l, \eta}\left(\left(\mathbb{R}^{d}\right)^{n-1}\right) \int_{A_{0}} \mathrm{e}^{-\psi^{l, \eta}(y)} \mathrm{d} y
\end{aligned}
$$


and the same result holds for $A_{1}$. This leads to the estimate:

$$
Q_{n}^{l, \eta}\left(\mathcal{F} m\left(A_{0}, A_{1}, \varepsilon, \delta\right)\right) \leqslant n \mathrm{e}^{-2 \bar{N} \underline{\varphi}} \nu_{n-1}^{l, \eta}\left(\left(\mathbb{R}^{d}\right)^{n-1}\right) \frac{41}{\delta} \exp \left(-\frac{\varepsilon^{2}}{5 \delta}\right) \int_{\mathbb{R}^{d}}\left(\mathbb{I}_{A_{0}}+\mathbb{I}_{A_{1}}\right) \mathrm{e}^{-\psi^{l, \eta}} \mathrm{d} y
$$

by summing over $n$ we obtain the desired result:

$$
\begin{aligned}
& \left.\left.Q_{z}^{l, \eta}\left(\mathcal{F} m\left(A_{0}, A_{1}, \varepsilon, \delta\right)\right)\right)=\frac{\mathrm{e}^{-z 2^{d} l^{d}}}{Z_{z}^{l, \eta}} \sum_{n=1}^{+\infty} \frac{z^{n}}{n !} Q_{n}^{l, \eta}\left(\mathcal{F} m\left(A_{0}, A_{1}, \varepsilon, \delta\right)\right) \cap\left(\mathbb{R}^{d}\right)^{n}\right) \\
& \quad \leqslant 41 \frac{\mathrm{e}^{-z 2^{d} l^{d}}}{Z_{z}^{l, \eta}} z\left(\sum_{n=1}^{+\infty} \frac{z^{n-1}}{(n-1) !} \nu_{n-1}^{l, \eta}\left(\left(\mathbb{R}^{d}\right)^{n-1}\right)\right) \mathrm{e}^{-2 \bar{N} \underline{\varphi}} \frac{1}{\delta} \exp \left(-\frac{\varepsilon^{2}}{5 \delta}\right) \int\left(\mathbb{I}_{A_{0}}+\mathbb{I}_{A_{1}}\right) \mathrm{e}^{-\psi^{l, \eta}} \mathrm{d} y \\
& \quad \leqslant\left(41 z \mathrm{e}^{-2 \bar{N} \underline{\varphi}}\right) \frac{1}{\delta} \exp \left(-\frac{\varepsilon^{2}}{5 \delta}\right) \int_{\mathbb{R}^{d}}\left(\mathbb{I}_{A_{0}}+\mathbb{I}_{A_{1}}\right) \mathrm{e}^{-\psi^{l, \eta}} \mathrm{d} y .
\end{aligned}
$$

Proof of Proposition 2.2. For $j$ in $\mathbb{N}$, let $a_{j}=K+\sqrt{\frac{\varepsilon^{2}}{\delta}+5 j}$. The sequence $\left(a_{j}\right)_{j}$ increases from $a_{0}=K+\frac{\varepsilon}{\sqrt{\delta}}$ to $+\infty$. Now for $Q=Q_{n}^{l, \eta}$ or $Q=Q_{z}^{l, \eta}$ consider

$$
\begin{aligned}
Q(\mathcal{F} m(K, \varepsilon, \delta))= & Q\left(\exists i,\left|X_{i}(0)\right| \leqslant K \text { and } w\left(X_{i}, \delta\right)>\varepsilon\right) \\
\leqslant \quad & Q\left(\exists i,\left|X_{i}(0)\right| \leqslant K \text { and } w\left(X_{i}, \delta\right)>\varepsilon \text { and }\left|X_{i}(1)\right| \leqslant a_{0}\right) \\
& +\sum_{j=0}^{+\infty} Q\left(\exists i,\left|X_{i}(0)\right| \leqslant K \text { and } a_{j}<\left|X_{i}(1)\right| \leqslant a_{j+1}\right)
\end{aligned}
$$

But $\left|X_{i}(0)\right| \leqslant K$ and $\left|X_{i}(1)\right|>a_{j}$ imply that $w\left(X_{i}, 1\right)>a_{j}-K$, so this is smaller than

$$
\begin{aligned}
\leqslant & Q\left(\exists i,\left|X_{i}(0)\right| \leqslant K,\left|X_{i}(1)\right| \leqslant a_{0} \text { and } w\left(X_{i}, \delta\right)>\varepsilon\right) \\
& +\sum_{j=0}^{+\infty} Q\left(\exists i,\left|X_{i}(0)\right| \leqslant K, a_{j}<\left|X_{i}(1)\right|<a_{j+1} \text { and } w\left(X_{i}, 1\right)>a_{j}-K\right)
\end{aligned}
$$

Using Lemma 2.1 we obtain:

$$
\begin{aligned}
Q(\mathcal{F} m(K, \varepsilon, \delta)) \leqslant & C(Q) \frac{1}{\delta} \exp \left(-\frac{\varepsilon^{2}}{5 \delta}\right) \int\left(\mathbb{1}_{B(0, K)}+\mathbb{I}_{B\left(0, a_{0}\right)}\right) \mathrm{e}^{-\psi^{l, \eta}} \mathrm{d} y \\
& +C(Q) \sum_{j=0}^{+\infty} \exp \left(-\frac{1}{5}\left(\frac{\varepsilon^{2}}{\delta}+5 j\right)\right) \int\left(\mathbb{I}_{B(0, K)}+\mathbb{I}_{B\left(0, a_{j+1}\right) \backslash B\left(0, a_{j}\right)}\right) \mathrm{e}^{-\psi^{l, \eta}} \mathrm{d} y \\
\leqslant & \frac{C(Q)}{\delta} \exp \left(-\frac{\varepsilon^{2}}{5 \delta}\right) \int\left(\mathbb{I}_{B(0, K)}+\mathbb{I}_{B\left(0, a_{0}\right)}+\sum_{j=0}^{+\infty} \mathrm{e}^{-j}\left(\mathbb{I}_{B(0, K)}+\mathbb{I}_{B\left(0, a_{j+1}\right) \backslash B\left(0, a_{j}\right)}\right)\right) \mathrm{e}^{-\psi^{l, \eta}} \mathrm{d} y
\end{aligned}
$$

with $C\left(Q_{n}^{l, \eta}\right)=41 n \mathrm{e}^{-2 \bar{N}} \underline{\varphi} \nu_{n-1}^{l, \eta}\left(\left(\mathbb{R}^{d}\right)^{n-1}\right)$ and $C\left(Q_{z}^{l, \eta}\right)=41 z \mathrm{e}^{-2 \bar{N}} \underline{\varphi}$.

Moreover, for $j \geqslant 1$ :

$$
\int_{B\left(0, a_{j+1}\right) \backslash B\left(0, a_{j}\right)} \mathrm{e}^{-\psi^{l, \eta}(y)} \mathrm{d} y \leqslant\left(a_{j+1}\right)^{d}|B(0,1)| \leqslant 3^{d} K^{d}|B(0,1)| \max \left(1, \frac{\varepsilon}{\sqrt{\delta}}\right)^{d} \sqrt{5(j+1)}^{d}
$$


and similarly:

$$
\int \mathbb{I}_{B\left(0, a_{0}\right)} \mathrm{e}^{-\psi^{l, \eta}(y)} \mathrm{d} y \leqslant\left(a_{0}\right)^{d}|B(0,1)| \leqslant 2^{d} K^{d}|B(0,1)| \max \left(1, \frac{\varepsilon}{\sqrt{\delta}}\right)^{d} .
$$

Since $\sum_{j=0}^{+\infty} \mathrm{e}^{-j} \leqslant 2$ and $\sum_{j=0}^{+\infty}(\sqrt{j+1})^{d} \mathrm{e}^{-j} \leqslant 2$ this leads to:

$$
Q(\mathcal{F} m(K, \varepsilon, \delta)) \leqslant C(Q) \frac{1}{\delta} \exp \left(-\frac{\varepsilon^{2}}{5 \delta}\right)|B(0, K)| 3 \times 3^{d} \max \left(1, \frac{\varepsilon}{\sqrt{\delta}}\right)^{d} \sqrt{5}^{d} .
$$

Finally

$$
\begin{aligned}
Q_{n}^{l, \eta}(\mathcal{F} m(K, \varepsilon, \delta)) & \leqslant n \mathrm{e}^{-2 \bar{N} \underline{\varphi}} \nu_{n-1}^{l, \eta}\left(\left(\mathbb{R}^{d}\right)^{n-1}\right) C_{d} \frac{1}{\delta} \exp \left(-\frac{\varepsilon^{2}}{6 \delta}\right) K^{d} \\
\text { and } \quad Q_{z}^{l, \eta}(\mathcal{F} m(K, \varepsilon, \delta)) & \leqslant z \mathrm{e}^{-2 \bar{N} \underline{\varphi}} C_{d} \frac{1}{\delta} \exp \left(-\frac{\varepsilon^{2}}{6 \delta}\right) K^{d}
\end{aligned}
$$

where $C_{d}=41|B(0,1)| 3 \times 3^{d} \sqrt{5}^{d} \sup _{x \in \mathbb{R}^{+}} \mathrm{e}^{-x^{2} / 5} \max (1, x)^{d} \mathrm{e}^{x^{2} / 6}$.

An alternative bound for $Q(\mathcal{F} m(K, \varepsilon, \delta))$ may be obtained using the fact that each indicator function is smaller than one:

$$
Q(\mathcal{F} m(K, \varepsilon, \delta)) \leqslant C(Q) \frac{1}{\delta} \exp \left(-\frac{\varepsilon^{2}}{5 \delta}\right) \int_{\mathbb{R}^{d}} 6 \mathrm{e}^{-\psi^{l, \eta}(y)} \mathrm{d} y .
$$

This completes the proof.

\subsection{Large chains of interacting particles}

Recall that two particles interact instantaneously only if the distance between their centers is smaller than $R$, the range of the potential $\varphi$. But more generally, a particle can have an influence on several ones during any small time interval. To modelize this, we introduce the notion of $(R+\varepsilon)$-chain of particles.

Definition 2.3. Let $x \in \mathcal{A}$ and $\varepsilon>0$. Each subset $\left\{x_{1}, \cdots, x_{n}\right\}$ of $x$ verifying $\left|x_{1}-x_{2}\right| \leqslant R+\varepsilon, \cdots, \mid x_{n-1}-$ $x_{n} \mid \leqslant R+\varepsilon$ is called an $(R+\varepsilon)$-chain of particles of $x$.

Now, let us fix $K \in \mathbb{N}^{*}, M \in \mathbb{N}^{*}$ and $\varepsilon>0$ and let $C h(K, M, R+\varepsilon)$ denote the event that there exists an $(R+\varepsilon)$-chain of $M$ particles with one end inside of $B(0, K)$, that is:

$C h(K, M, R+\varepsilon)=\left\{x \in \mathcal{A}, \exists\left\{x_{1}, \cdots, x_{M}\right\}\right.$ subset of $x,\left|x_{1}\right|<K$ and $\left.\left|x_{1}-x_{2}\right| \leqslant R+\varepsilon, \cdots,\left|x_{M-1}-x_{M}\right| \leqslant R+\varepsilon\right\}$.

Our aim in this subsection is to estimate the $\mu_{z}^{l, \eta}$-probability that such a chain exists.

Proposition 2.4. For every $M \in \mathbb{N}^{*}, \varepsilon>0, l \in \mathbb{N}^{*}, \eta \in \mathcal{A}$ and $K \in \mathbb{R}^{+}$, we have:

$$
\mu_{z}^{l, \eta}\left(\bigcup_{K=1}^{+\infty} C h(K, M, R+\varepsilon)\right) \leqslant\left(z|B(0,1)| \exp (-2 \bar{N} \underline{\varphi})\left((R+\varepsilon)^{d}-r^{d}\right)\right)^{M-1} .
$$

From this proposition, we easily deduce the following corollary used in Section 2.4. 
Corollary 2.5. There exists a critical activity $z_{c}=\frac{\exp (2 \bar{N} \underline{\varphi})}{\left(R^{d}-r^{d}\right)|B(0,1)|}$ such that for each $\left.z>0, \varepsilon \in\right] 0,1[$ and $M \in \mathbb{N}^{*}$,

$$
\sup _{l \in \mathbb{N}^{*}} \sup _{\eta \in \mathcal{A}} \mu_{z}^{l, \eta}\left(\bigcup_{K=1}^{+\infty} C h(K, M, R+\varepsilon)\right) \leqslant\left(\frac{z}{z_{c}} \frac{(R+\varepsilon)^{d}-r^{d}}{R^{d}-r^{d}}\right)^{M-1} .
$$

In particular, for any $z<z_{c}$ there exists $\varepsilon>0$ such that

$$
\lim _{M \rightarrow+\infty} \sup _{l \in \mathbb{N}^{*}} \sup _{\eta \in \mathcal{A}} \mu_{z}^{l, \eta}\left(\bigcup_{K=1}^{+\infty} C h(K, M, R+\varepsilon)\right)=0
$$

Proof of Proposition 2.4. Each configuration in $\left(\mathbb{R}^{d}\right)^{n} \cap C h(K, M, R+\varepsilon)$ has exactly $\frac{n !}{(n-M) !}$ representants in $\left(\mathbb{R}^{d}\right)^{n}$ such that $\left(x_{n-M+1}, \ldots, x_{n}\right)$ is a fixed $M$-uple verifying $\left|x_{n-M+1}\right|<K$ and $\left|x_{n-M+1}-x_{n-M+2}\right| \leqslant R+$ $\varepsilon, \cdots,\left|x_{n-1}-x_{n}\right| \leqslant R+\varepsilon$. Since $\beta_{n}^{l, \eta}\left(x_{1}, \ldots, x_{n}\right)$ and $\mathbb{I}_{\mathcal{A}}\left(x_{1}, \ldots, x_{n}\right)$ do not change by permutation of the $x_{i}$ 's, this leads to:

$$
\begin{aligned}
& \nu_{n}^{l, \eta}(C h(K, M, R+\varepsilon)) \\
& =\frac{n !}{(n-M) !} \int_{\left(\mathbb{R}^{d}\right)^{n}} \mathbb{I}_{\left|x_{n-M+1}\right|<K} \mathbb{I}_{\mathcal{A}}\left(x_{1}, \ldots, x_{n}\right) \mathrm{e}^{-\beta_{n}^{l, \eta}\left(x_{1}, \ldots, x_{n}\right)} \prod_{i=n-M+1}^{n-1} \mathbb{I}_{\left|x_{i}-x_{i+1}\right| \leqslant R+\varepsilon} \mathrm{d} x_{1} \cdots \mathrm{d} x_{n} .
\end{aligned}
$$

Remarking that

$$
\mathbb{I}_{\mathcal{A}}\left(x_{1}, \ldots, x_{n}\right) \leqslant \mathbb{1}_{\left|x_{n}-x_{n-1}\right| \geqslant r} \mathbb{1}_{\mathcal{A}}\left(x_{1}, \ldots, x_{n-1}\right),
$$

using again inequality (8) and integrating with respect to $x_{n}$ we obtain:

$$
\begin{aligned}
& \nu_{n}^{l, \eta}(C h(K, M, R+\varepsilon)) \\
& \quad \leqslant n \frac{(n-1) !}{((n-1)-(M-1)) !} \int_{\left(\mathbb{R}^{d}\right)^{n}} \mathbb{I}_{\left|x_{n-M+1}\right|<K}\left(\prod_{i=n-M+1}^{n-2} \mathbb{1}_{r \leqslant\left|x_{i}-x_{i+1}\right| \leqslant R+\varepsilon}\right) \mathbb{I}_{\mathcal{A}}\left(x_{1}, \ldots, x_{n-1}\right) \\
& \quad \leqslant n \mathrm{e}^{-2 \bar{N} \underline{\varphi}}\left((R+\varepsilon)^{d}-r^{d}\right)|B(0,1)| \underline{\mathbb{I}}_{r \leqslant\left|x_{n}-x_{n-1}\right| \leqslant R+\varepsilon} \mathrm{e}^{-2 \bar{N} \underline{\varphi}} \mathrm{e}^{-\beta_{n-1}^{l, \eta}\left(x_{1}, \ldots, x_{n-1}\right)} \mathrm{d} x_{1} \cdots \mathrm{d} x_{n} \\
& \quad K, M-1, R+\varepsilon)) .
\end{aligned}
$$

By definition of $\mu_{z}^{l, \eta}$ (see (5))

$$
\mu_{z}^{l, \eta}(C h(K, M, R+\varepsilon))=\frac{\mathrm{e}^{-z 2^{d} l^{d}}}{Z_{z}^{l, \eta}} \sum_{n \geqslant M} \frac{z^{n}}{n !} \nu_{n}^{l, \eta}(C h(K, M, R+\varepsilon)) .
$$

Using the above inequality and iterating the result on $M$, we obtain:

$$
\begin{aligned}
& \mu_{z}^{l, \eta}(C h(K, M, R+\varepsilon)) \\
& \quad \leqslant \mathrm{e}^{-2 \bar{N} \underline{\varphi}}\left((R+\varepsilon)^{d}-r^{d}\right)|B(0,1)| \frac{\mathrm{e}^{-z 2^{d} l^{d}}}{Z_{z}^{l, \eta}} z \sum_{n \geqslant M} \frac{z^{n-1}}{(n-1) !} \nu_{n-1}^{l, \eta}(C h(K, M-1, R+\varepsilon) \\
& \quad \leqslant z \mathrm{e}^{-2 \bar{N} \underline{\varphi}}\left((R+\varepsilon)^{d}-r^{d}\right)|B(0,1)| \mu_{z}^{l, \eta}(C h(K, M-1, R+\varepsilon)) \\
& \quad \leqslant\left(z \mathrm{e}^{-2 \bar{N} \underline{\varphi}}\left((R+\varepsilon)^{d}-r^{d}\right)|B(0,1)|\right)^{M-1} .
\end{aligned}
$$

Since the event $C h(K, M, R+\varepsilon)$ increases as a function of $K$

$$
\mu_{z}^{l, \eta}\left(\bigcup_{K=1}^{+\infty} C h(K, M, R+\varepsilon)\right) \leqslant\left(z|B(0,1)| \exp (-2 \bar{N} \underline{\varphi})\left((R+\varepsilon)^{d}-r^{d}\right)\right)^{M-1}
$$




\subsection{Estimates on the set of regular paths}

Let $\mathcal{B}(m, a, \varepsilon)$ denote the following set of bad paths, in which either a particle has a high oscillation in a small time interval or belongs to a large chain of interacting particles:

$$
\begin{array}{rl}
\forall m \in \mathbb{N}^{*} \forall a \geqslant 1 \quad \forall \varepsilon>0 & \mathcal{B}(m, a, \varepsilon)=\tilde{\mathcal{B}}(m, a, \varepsilon) \cup \tilde{\tilde{\mathcal{B}}}(m, \varepsilon) \\
\text { where } \tilde{\mathcal{B}}(m, a, \varepsilon)=\left\{X \in \mathcal{C}([0,1], \mathcal{A}), \exists i, w\left(X_{i}, \frac{1}{m}\right)>\frac{\varepsilon}{4} \text { and } \exists t \leqslant 1,\left|X_{i}(t)\right| \leqslant a+2 m^{2}\right\} \\
\begin{aligned}
\exists k \in\{0, \ldots, m-1\}, \text { there exists an } \\
\text { and } \left.\quad \begin{array}{l}
(R+\varepsilon)-\text { chain of particles of } X\left(\frac{k}{m}\right) \\
\text { with diameter greater than } m-\varepsilon)
\end{array}\right\}
\end{aligned}
\end{array}
$$

Let us remark that $a \mapsto \tilde{\mathcal{B}}(m, a, \varepsilon)$ is non-decreasing but $\tilde{\mathcal{B}}(m, a, \varepsilon)$ is not monotone as a function of $m$ and $\varepsilon$. Our aim in this section is to estimate the probability of $\mathcal{B}(m, a, \varepsilon)$ under $Q_{z}^{l, \eta}$.

Proposition 2.6. For each $m \in \mathbb{N}^{*}$ and $a \geqslant 1$ :

$$
\sup _{l \in \mathbb{N} *} \sup _{\eta \in \mathcal{A}} Q_{z}^{l, \eta}(\tilde{\mathcal{B}}(m, a, \varepsilon)) \leqslant z C_{d}^{\prime} \mathrm{e}^{-2 \bar{N} \underline{\varphi}} a^{d} m^{2 d} \exp \left(-\frac{\varepsilon^{2}}{96} m\right)
$$

where the constant $C_{d}^{\prime}$ only depends on dimension $d$. One also has, for $m \in \mathbb{N}^{*}$ :

$$
\sup _{l \in \mathbb{N}^{*}} \sup _{\eta \in \mathcal{A}} Q_{z}^{l, \eta}(\tilde{\tilde{\mathcal{B}}}(m, \varepsilon)) \leqslant m\left(z|B(0,1)| \exp (-2 \bar{N} \underline{\varphi})\left((R+\varepsilon)^{d}-r^{d}\right)\right)^{\left[\frac{m}{R+\varepsilon}\right]}
$$

If $z<z_{c}$ and $\varepsilon$ is small enough (depending on $z$ ), this implies that the left hand side decreases exponentially fast as a function of $m$.

Proof of Proposition 2.6.

$$
\begin{aligned}
Q_{z}^{l, \eta}(\tilde{\mathcal{B}}(m, a, \varepsilon)) \leqslant & Q_{z}^{l, \eta}\left(\exists i, w\left(X_{i}, \frac{1}{m}\right)>\frac{\varepsilon}{4} \text { and }\left|X_{i}(0)\right| \leqslant a+3 m^{2}\right) \\
& +Q_{z}^{l, \eta}\left(\exists i,\left|X_{i}(0)\right|>a+3 m^{2} \text { and } \exists t \leqslant 1,\left|X_{i}(t)\right| \leqslant a+2 m^{2}\right)
\end{aligned}
$$

The second term of the sum is smaller than

$$
\sum_{j=1}^{+\infty} Q_{z}^{l, \eta}\left(\exists i, a+(2+j) m^{2}<\left|X_{i}(0)\right| \leqslant a+(3+j) m^{2} \text { and } w\left(X_{i}, 1\right)>j m^{2}\right)
$$


Thus using Proposition 2.2, we obtain:

$$
\begin{aligned}
Q_{z}^{l, \eta}(\tilde{\mathcal{B}}(m, a, \varepsilon)) \leqslant & Q_{z}^{l, \eta}\left(\mathcal{F} m\left(a+3 m^{2}, \frac{\varepsilon}{4}, \frac{1}{m}\right)\right)+\sum_{j=1}^{+\infty} Q_{z}^{l, \eta}\left(\mathcal{F} m\left(a+(3+j) m^{2}, j m^{2}, 1\right)\right) \\
\leqslant & z C_{d} \mathrm{e}^{-2 \bar{N} \underline{\varphi}} m \exp \left(-\frac{\varepsilon^{2}}{96} m\right)\left(a+3 m^{2}\right)^{d} \\
& \quad+\sum_{j=1}^{+\infty} z C_{d} \mathrm{e}^{-2 \bar{N} \underline{\varphi}} \exp \left(-\frac{j^{2} m^{4}}{6}\right)\left(a+(3+j) m^{2}\right)^{d} \\
\leqslant & z C_{d} \mathrm{e}^{-2 \bar{N} \underline{\varphi}}\left(m \exp \left(-\frac{\varepsilon^{2}}{96} m\right)\left(a+3 m^{2}\right)^{d}+\sum_{j=1}^{+\infty} \exp \left(-\frac{j^{2} m^{4}}{6}\right)\left(a+(3+j) m^{2}\right)^{d}\right) \\
\leqslant & z C_{d} \mathrm{e}^{-2 \bar{N} \underline{\varphi}} 8^{d} a^{d} m^{2 d}\left(\exp \left(-\frac{\varepsilon^{2}}{96} m\right)+\sum_{j=1}^{+\infty} \exp \left(-\frac{j^{2} m^{4}}{6}\right) j^{d}\right) \\
\leqslant & z C_{d} \mathrm{e}^{-2 \bar{N} \underline{\varphi}} 8^{d} a^{d} m^{2 d} \exp \left(-\frac{\varepsilon^{2}}{96} m\right)\left(1+\sum_{j=1}^{+\infty} j^{d} \exp \left(-\frac{j^{2}}{12}\right)\right) .
\end{aligned}
$$

Defining the constant $C_{d}^{\prime}$ equal to $C_{d} 8^{d}\left(1+\sum_{j=1}^{+\infty} j^{d} \exp \left(-\frac{j^{2}}{12}\right)\right)<+\infty$ we obtain

$$
Q_{z}^{l, \eta}(\tilde{\mathcal{B}}(m, a, \varepsilon)) \leqslant z C_{d}^{\prime} \mathrm{e}^{-2 \bar{N} \underline{\varphi}} a^{d} m^{2 d} \exp \left(-\frac{\varepsilon^{2}}{96} m\right) .
$$

We now have to find a similar estimate for

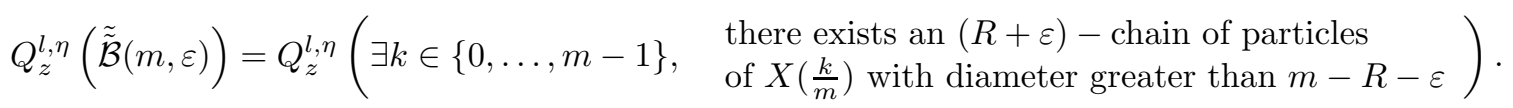

Thanks to the stationarity of $Q_{z}^{l, \eta}$, this probability is smaller than

$$
\sum_{k=0}^{m-1} \mu_{z}^{l, \eta}\left(x \in \mathcal{A}, \quad \begin{array}{l}
\text { there exists an }(R+\varepsilon)-\text { chain of particles } \\
\text { of } x \text { with diameter greater than } m-R-\varepsilon
\end{array}\right) .
$$

It is necessary to have at least $\left[\frac{m}{R+\varepsilon}\right]+1$ particles $([x]$ denotes the integer part of $x)$ to construct a chain of length greater than $m-R-\varepsilon$ where each particle is at distance smaller than $(R+\varepsilon)$ from its neighbors. Thus the above quantity is smaller than $m \mu_{z}^{l, \eta}\left(\bigcup_{K=1}^{+\infty} C h\left(K,\left[\frac{m}{R+\varepsilon}\right]+1, R+\varepsilon\right)\right)$. Due to Proposition 2.4 , this is bounded from above by

$$
m\left(z|B(0,1)| \exp (-2 \bar{N} \underline{\varphi})\left((R+\varepsilon)^{d}-r^{d}\right)\right)^{\left[\frac{m}{R+\varepsilon}\right]} .
$$

\section{Convergence of the Approximations}

The aim of this section is to prove the convergence of the sequence $\left(X^{l, x}\right)_{l}$ to a limit process $X^{\infty, x}$. We shall check in the next section that $X^{\infty, x}$ is a solution of $(\mathcal{E})$.

We fix $x \in \mathcal{A}$. As usual for infinite-dimensional stochastic equations, we study $X^{l, x}(\omega)$ for each $\omega$ in a set $\Omega_{0}^{x} \subset \Omega$ and prove that the set $\Omega \backslash \Omega_{0}^{x}$ is negligible.

For each $\rho \in \mathbb{N}^{*}$ and $l \geqslant \rho-r+1$, let $m(\rho, l)$ and $a(\rho, l)$ denote the following integers:

$$
m(\rho, l)=[\sqrt{l-\rho-r}]-1 \quad \text { and } \quad a(\rho, l)=\rho+m(\rho, l) .
$$


Remark that

and

$$
a(\rho, l)+m(\rho, l)^{2}+1<l-r
$$

$$
\forall C>0 \quad \sum_{l} a(\rho, l)^{d} m(\rho, l)^{2 d} \mathrm{e}^{-C m(\rho, l)}<+\infty .
$$

Let $1 / \mathbb{N}$ denote the set $\{1,1 / 2,1 / 3, \cdots\}$ of real numbers $\varepsilon$ such that $1 / \varepsilon \in \mathbb{N}$. Let us now define the set $\Omega_{0}^{x}$ as follows:

$$
\begin{aligned}
\Omega_{0}^{x}= & \left\{\omega \in \Omega \text { s.t. } \exists \varepsilon_{0} \in 1 / \mathbb{N} \forall \varepsilon \leqslant \varepsilon_{0} \forall \rho \in \mathbb{N}^{*} \exists l_{0} \in \mathbb{N}^{*}, \forall l \geqslant l_{0}\right. \\
& \left.X^{l, x}(\omega, \cdot) \notin \mathcal{B}(m(\rho, l), a(\rho, l), \varepsilon) \text { and } X^{l+1, x}(\omega, \cdot) \notin \mathcal{B}(m(\rho, l), a(\rho, l), \varepsilon)\right\} \\
= & \liminf _{\varepsilon \rightarrow 0} \bigcap_{\rho \in \mathbb{N}^{*}} \liminf _{l \rightarrow+\infty}\left\{X^{l, x} \notin \mathcal{B}(m(\rho, l), a(\rho, l), \varepsilon)\right\} \cap\left\{X^{l+1, x} \notin \mathcal{B}(m(\rho, l), a(\rho, l), \varepsilon)\right\} .
\end{aligned}
$$

Proposition 3.1. For every $x \in \mathcal{A}$, every $\omega$ in $\Omega_{0}^{x}$ and every $i \in \mathbb{N}$, the sequence of processes $\left(X_{i}^{l, x}(\omega, t), L_{i j}^{l, x}(\omega, t)\right.$, $j \in \mathbb{N}, t \in[0,1])_{l \in \mathbb{N}^{*}}$ elements of $\mathcal{C}\left([0,1], \mathbb{R}^{d} \times \mathbb{R}_{+}^{\mathbb{N}}\right)$ converges in the sense of uniform convergence of continuous paths to a limit denoted by $\left(X_{i}^{\infty, x}(\omega, t), L_{i j}^{\infty, x}(\omega, t), j \in \mathbb{N}, t \in[0,1]\right)$. Moreover, this sequence is stationary: for any $x \in \mathcal{A}, \omega \in \Omega_{0}^{x}, i \in \mathbb{N}$

$$
\exists l_{0} \quad \forall l \geqslant l_{0}, X_{i}^{l, x}(\omega, \cdot) \equiv X_{i}^{\infty, x}(\omega, \cdot) \text { on }[0,1], \text { and }, \forall j \in \mathbb{N} L_{i j}^{l, x}(\omega, \cdot) \equiv L_{i j}^{\infty, x}(\omega, \cdot) \text { on }[0,1] .
$$

Proposition 3.2. For each Gibbs measure $\mu \in \mathcal{G}(z)$ with $z<z_{c}$ one has $\int_{\mathcal{M}} P\left(\Omega_{0}^{x}\right) \mathrm{d} \mu(x)=1$. This means that defining $\underline{\mathcal{A}}$ by $\underline{\mathcal{A}}=\left\{x \in \mathcal{A}, P\left(\Omega_{0}^{x}\right)=1\right\}$, one has

$$
\forall z<z_{c} \quad \forall \mu \in \mathcal{G}(z) \quad \mu(\underline{\mathcal{A}})=1 .
$$

Proof of Proposition 3.2. Let us fix $\mu \in \mathcal{G}(z)$. We want to prove that $\int_{\mathcal{A}} P\left(\Omega \backslash \Omega_{0}^{x}\right) \mathrm{d} \mu(x)=0$. By definition of $\Omega_{0}^{x}$

$$
\begin{aligned}
P\left(\Omega \backslash \Omega_{0}^{x}\right)=P \quad\left(\forall \varepsilon_{0} \in 1 / \mathbb{N}, \exists \varepsilon \leqslant \varepsilon_{0} \exists \rho \in \mathbb{N}^{*} \forall l_{0} \in \mathbb{N}^{*} \exists l \geqslant l_{0}, \begin{array}{l}
X^{l, x} \in \mathcal{B}(m(\rho, l), a(\rho, l), \varepsilon) \text { or } \\
X^{l, x} \in \mathcal{B}(m(\rho, l-1), a(\rho, l-1), \varepsilon)
\end{array}\right) \\
\leqslant \sum_{\varepsilon \leqslant \varepsilon_{0}} \sum_{\rho \in \mathbb{N}^{*}} P\left(\limsup _{l \rightarrow+\infty}\left\{X^{l, x} \in \mathcal{B}(m(\rho, l), a(\rho, l), \varepsilon) \cup \mathcal{B}(m(\rho, l-1), a(\rho, l-1), \varepsilon)\right\}\right)
\end{aligned}
$$

for all $\varepsilon_{0} \in 1 / \mathbb{N}$. Thanks to Borel-Cantelli lemma, this vanishes as soon as there exists $\varepsilon_{0} \in 1 / \mathbb{N}$ such that for all $\varepsilon \leqslant \varepsilon_{0}$ and $\rho \in \mathbb{N}^{*}$

$$
\sum_{l=\rho+2}^{+\infty} \int_{\mathcal{A}} P\left(X^{l, x} \in \mathcal{B}(m(\rho, l), a(\rho, l), \varepsilon) \cup \mathcal{B}(m(\rho, l-1), a(\rho, l-1), \varepsilon)\right) \mathrm{d} \mu(x)<+\infty .
$$

We shall show (step 1 ) that for $l \in \mathbb{N}^{*}$ and $\Lambda=[-l, l]^{d}$, the following inequalities hold:

$$
\begin{aligned}
& \sup _{\Theta \in \mathcal{F}_{1}}\left|\int_{\mathcal{A}} P\left(X^{l, x} \in \Theta\right) \mathrm{d} \mu(x)-\int_{\mathcal{A}} Q_{z}^{l, \eta}(\Theta) \mathrm{d} \mu(\eta)\right| \leqslant \int_{\mathcal{A}} \sup _{\|f\| \leqslant 1}\left|\int_{\mathcal{A}} f \mathrm{~d} \mu\left(. \mid \eta_{\left([-l, l]^{d}\right)^{c}}\right)-\int_{\mathcal{A}} f \mathrm{~d} \mu_{z}^{l, \eta}\right| \mathrm{d} \mu(\eta) \\
& \quad \forall \eta \in \mathcal{A} \quad \sup _{\|f\| \leqslant 1}\left|\int_{\mathcal{A}} f(x) \mathrm{d} \mu\left(x \mid \eta_{\left([-l, l]^{d}\right)^{c}}\right)-\int_{\mathcal{A}} f(x) \mathrm{d} \mu_{z}^{l, \eta}(x)\right| \leqslant 2\left(1-\frac{Z_{z}^{\Lambda, \eta}}{Z_{z}^{l, \eta}}\right)
\end{aligned}
$$


and (step 2) that

$$
\forall \eta \in \mathcal{A} \quad 0 \leqslant 1-\frac{Z_{z}^{\Lambda, \eta}}{Z_{z}^{l, \eta}} \leqslant z \exp (-2 \bar{N} \underline{\varphi}) \int_{\mathbb{R}^{d}} \mathbb{I}_{\psi^{l, \eta}(y)>0} \exp \left(-\psi^{l, \eta}(y)\right) \mathrm{d} y
$$

Inequality (18) and assumption (3) on $\psi^{l, \eta}$ imply that

$$
\sum_{l=1}^{+\infty} \int_{\mathcal{A}}\left(1-\frac{Z_{z}^{\Lambda, \eta}}{Z_{z}^{l, \eta}}\right) \mathrm{d} \mu(\eta)<+\infty
$$

Then for each $\rho$ and $l$ fixed, we choose $\Theta=\mathcal{B}(m(\rho, l), a(\rho, l), \varepsilon) \cup \mathcal{B}(m(\rho, l-1), a(\rho, l-1), \varepsilon)$. Thanks to $(17)$ and (18), in order to prove (16), it suffices to prove that there exists $\varepsilon_{0} \in 1 / \mathbb{N}$ such that for all $\varepsilon<\varepsilon_{0}$ and $\rho \in \mathbb{N}^{*}$

$$
\begin{aligned}
& \sum_{l=\rho+2}^{+\infty} \int Q_{z}^{l, \eta}(\tilde{\mathcal{B}}(m(\rho, l), a(\rho, l), \varepsilon) \cup \tilde{\mathcal{B}}(m(\rho, l-1), a(\rho, l-1), \varepsilon)) \mathrm{d} \mu(\eta) \\
& \quad+\sum_{l=\rho+2}^{+\infty} \int Q_{z}^{l, \eta}(\tilde{\tilde{\mathcal{B}}}(m(\rho, l), \varepsilon) \cup \tilde{\tilde{\mathcal{B}}}(m(\rho, l-1), \varepsilon)) \mathrm{d} \mu(\eta)<+\infty
\end{aligned}
$$

By proposition 2.6, this is smaller than

$$
\begin{gathered}
\int z C_{d}^{\prime} \mathrm{e}^{-2 \bar{N} \underline{\varphi}} \sum_{l=\rho+2}^{+\infty} a(\rho, l)^{d} m(\rho, l)^{2 d} \exp \left(-\frac{\varepsilon^{2}}{96} m(\rho, l)\right) \\
+a(\rho, l-1)^{d} m(\rho, l-1)^{2 d} \exp \left(-\frac{\varepsilon^{2}}{96} m(\rho, l-1)\right) \mathrm{d} \mu(\eta) \\
+\int \sum_{l=\rho+2}^{+\infty} m(\rho, l)\left(z|B(0,1)| \exp (-2 \bar{N} \underline{\varphi})\left((R+\varepsilon)^{d}-r^{d}\right)\right)^{\left[\frac{m(\rho, l)}{R+\varepsilon}\right]} \\
+m(\rho, l-1)\left(z|B(0,1)| \exp (-2 \bar{N} \underline{\varphi})\left((R+\varepsilon)^{d}-r^{d}\right)\right)^{\left[\frac{m(\rho, l-1)}{R+\varepsilon}\right]} \mathrm{d} \mu(\eta) .
\end{gathered}
$$

Thus we only have to prove that

$$
\begin{aligned}
& \exists \varepsilon_{0} \in 1 / \mathbb{N} \quad \forall \varepsilon<\varepsilon_{0} \quad \forall \rho \in \mathbb{N}^{*} \sum_{l=\rho+2}^{+\infty} a(\rho, l)^{d} m(\rho, l)^{2 d} \exp \left(-\frac{\varepsilon^{2}}{96} m(\rho, l)\right)<+\infty \\
& \text { and } \quad \sum_{l=\rho+2}^{+\infty} m(\rho, l)\left(z|B(0,1)| \exp (-2 \bar{N} \underline{\varphi})\left((R+\varepsilon)^{d}-r^{d}\right)\right)^{\left[\frac{m(\rho, l)}{R+\varepsilon}\right]}<+\infty .
\end{aligned}
$$

The first series converges for each $\varepsilon \in 1 / \mathbb{N}$ and each $\rho \in \mathbb{N}^{*}$ thanks to (14). The second one also converges thanks to (14), as soon as $\left(z|B(0,1)| \exp (-2 \bar{N} \underline{\varphi})\left(\left(R+\varepsilon_{0}\right)^{d}-r^{d}\right)\right)<1$, which is true for $\varepsilon_{0}$ small enough when $z<z_{c}$. It remains to prove (17) and (18). 
Step 1. Proof of (17)

Let us fix $l \in \mathbb{N}^{*}$ and $\Lambda=[-l, l]^{d}$. For each event $\Theta$ on $\mathcal{C}([0,1])$, by definition of $Q_{z}^{l, \eta}$ :

$$
\begin{aligned}
& \int_{\mathcal{A}} P\left(X^{l, x} \in \Theta\right) \mathrm{d} \mu(x)-\int_{\mathcal{A}} Q_{z}^{l, \eta}(\Theta) \mathrm{d} \mu(\eta) \\
& \leqslant \int_{\mathcal{A}} \int_{\mathcal{A}} P\left(X^{l, x \eta_{\Lambda^{c}}} \in \Theta\right) \mathrm{d} \mu\left(x \mid \eta_{\Lambda^{c}}\right) \mathrm{d} \mu(\eta)-\int_{\mathcal{A}} \int_{\mathcal{A}} P\left(X^{l, \eta, \operatorname{Card}(\mathrm{x})}(x, \cdot) \in \Theta\right) \mathrm{d} \mu_{z}^{l, \eta}(x) \mathrm{d} \mu(\eta) .
\end{aligned}
$$

If $x \eta \in \mathcal{A}$ then $P\left(X^{l, \eta, \operatorname{Card}(\mathrm{x})}(x, \cdot) \in \Theta\right)=P\left(X^{l, x, \eta_{\Lambda^{c}}} \in \Theta\right)$ and since this quantity is smaller than 1 , we obtain:

$$
\left|\int_{\mathcal{A}} P\left(X^{l, x} \in \Theta\right) \mathrm{d} \mu(x)-\int_{\mathcal{A}} Q_{z}^{l, \eta}(\Theta) \mathrm{d} \mu(\eta)\right| \leqslant \int_{\mathcal{A}} \sup _{\|f\| \leqslant 1}\left|\int_{\mathcal{A}} f(x) \mathrm{d} \mu\left(x \mid \eta_{\Lambda^{c}}\right)-\int_{\mathcal{A}} f(x) \mathrm{d} \mu_{z}^{l, \eta}(x)\right| \mathrm{d} \mu(\eta) .
$$

Since $\mu \in \mathcal{G}(z)$, using the conditional density of $\mu$ with respect to $\pi^{z}$ and the definition of $\mu_{z}^{l, \eta}$, one has for each $f: \mathcal{A} \rightarrow \mathbb{R}$ bounded by $1:$

$$
\begin{aligned}
& \left|\int_{\mathcal{A}} f(x) \mathrm{d} \mu\left(x \mid \eta_{\Lambda^{c}}\right)-\int_{\mathcal{A}} f(x) \mathrm{d} \mu_{z}^{l, \eta}(x)\right| \\
& =\mid \frac{\mathrm{e}^{-z|\Lambda|}}{Z_{z}^{\Lambda, \eta}}\left[f\left(\eta_{\Lambda^{c}}\right)+\sum_{n=1}^{+\infty} \frac{z^{n}}{n !} \int_{\Lambda^{n}} f\left(y \eta_{\Lambda^{c}}\right) \mathbb{I}_{\mathcal{A}}\left(y \eta_{\Lambda^{c}}\right) \exp \left(-\sum_{1 \leqslant i<j \leqslant n} \varphi\left(y_{i}-y_{j}\right)-\sum_{\substack{1 \leqslant i \leqslant n \\
\eta_{j} \in \Lambda^{c}}} \varphi\left(y_{i}-\eta_{j}\right)\right) \mathrm{d} y\right] \\
& -\frac{\mathrm{e}^{-z 2^{d} l^{d}}}{Z_{z}^{l, \eta}}\left[f\left(\eta_{\Lambda^{c}}\right)+\sum_{n=1}^{+\infty} \frac{z^{n}}{n !} \int_{\left(\mathbb{R}^{d}\right)^{n}} f\left(y \eta_{\Lambda^{c}}\right) \mathrm{e}^{-\beta_{n}^{l, \eta}(y)} \mathbb{I}_{\mathcal{A}}(y) \mathrm{d} y\right] \mid .
\end{aligned}
$$

Note that $\beta_{n}^{l, \eta}(y)=\sum_{1 \leqslant i<j \leqslant n} \varphi\left(y_{i}-y_{j}\right)+\sum_{\substack{1 \leqslant i \leqslant n \\ \eta_{j} \in \Lambda^{c}}} \varphi\left(y_{i}-\eta_{j}\right)$ for any $y \in \Lambda^{n}$ verifying $y \eta_{\Lambda^{c}} \in \mathcal{A}$, because $\psi^{l, \eta}\left(y_{i}\right)=0$ for each $i$ in this case. Thus the above quantity is equal to

$$
\begin{aligned}
\mid f\left(\eta_{\Lambda^{c}}\right) & {\left[\frac{\mathrm{e}^{-z|\Lambda|}}{Z_{z}^{\Lambda, \eta}}-\frac{\mathrm{e}^{-z 2^{d} l^{d}}}{Z_{z}^{l, \eta}}\right]+\sum_{n=1}^{+\infty} \frac{z^{n}}{n !}\left[\frac{\mathrm{e}^{-z|\Lambda|}}{Z_{z}^{\Lambda, \eta}}-\frac{\mathrm{e}^{-z 2^{d} l^{d}}}{Z_{z}^{l, \eta}}\right] \int_{\Lambda^{n}} f\left(y \eta_{\Lambda^{c}}\right) \mathbb{I}_{\mathcal{A}}\left(y \eta_{\Lambda^{c}}\right) \mathrm{e}^{-\beta_{n}^{l, \eta}(y)} \mathrm{d} y } \\
& -\frac{\mathrm{e}^{-z 2^{d} l^{d}}}{Z_{z}^{l, \eta}} \sum_{n=1}^{+\infty} \frac{z^{n}}{n !} \int_{\left(\mathbb{R}^{d}\right)^{n}} f\left(y \eta_{\Lambda^{c}}\right) \mathrm{e}^{-\beta_{n}^{l, \eta}(y)}\left(\mathbb{I}_{\mathcal{A}}(y)-\mathbb{I}_{\mathcal{A}}\left(y \eta_{\Lambda^{c}}\right) \mathbb{I}_{\Lambda^{n}}(y)\right) \mathrm{d} y \mid .
\end{aligned}
$$

Recall $\mathrm{e}^{-z|\Lambda|}=\mathrm{e}^{-z 2^{d} l^{d}}$ and

$$
\begin{aligned}
Z_{z}^{\Lambda, \eta} & =\mathrm{e}^{-z|\Lambda|}\left[1+\sum_{n=1}^{+\infty} \frac{z^{n}}{n !} \int_{\Lambda^{n}} \mathbb{I}_{\mathcal{A}}\left(y \eta_{\Lambda^{c}}\right) \mathrm{e}^{-\beta_{n}^{l, \eta}(y)} \mathrm{d} y\right] \\
& \leqslant \mathrm{e}^{-z 2^{d} l^{d}}\left[1+\sum_{n=1}^{+\infty} \frac{z^{n}}{n !} \int_{\left(\mathbb{R}^{d}\right)^{n}} \mathbb{I}_{\mathcal{A}}(y) \mathrm{e}^{-\beta_{n}^{l, \eta}(y)} \mathrm{d} y\right]=Z_{z}^{l, \eta} .
\end{aligned}
$$

We then obtain:

$$
\begin{aligned}
& \left|\int_{\mathcal{A}} f(x) \mathrm{d} \mu\left(x \mid \eta_{\Lambda^{c}}\right)-\int_{\mathcal{A}} f(x) \mathrm{d} \mu_{z}^{l, \eta}(x)\right| \\
& \leqslant\left|\frac{\mathrm{e}^{-z|\Lambda|}}{Z_{z}^{\Lambda, \eta}}-\frac{\mathrm{e}^{-z 2^{d} l^{d}}}{Z_{z}^{l, \eta}}\right| \mathrm{e}^{z|\Lambda|} Z_{z}^{\Lambda, \eta}+\frac{\mathrm{e}^{-z 2^{d} l^{d}}}{Z_{z}^{l, \eta}}\left|\mathrm{e}^{z 2^{d} l^{d}} Z_{z}^{l, \eta}-\mathrm{e}^{z|\Lambda|} Z_{z}^{\Lambda, \eta}\right|=2\left(1-\frac{Z_{z}^{\Lambda, \eta}}{Z_{z}^{l, \eta}}\right)
\end{aligned}
$$

and (17) is proven. 
Step 2. Proof of (18)

This final step of the proof of Proposition 3.2 is straightforward, simply using the definitions of $Z_{z}^{l, \eta}, Z_{z}^{\Lambda, \eta}$ and $\psi^{l, \eta}$ :

$$
\begin{aligned}
1-\frac{Z_{z}^{\Lambda, \eta}}{Z_{z}^{l, \eta}}= & \frac{\mathrm{e}^{-z 2^{d} l^{d}}}{Z_{z}^{l, \eta}} \sum_{n=0}^{+\infty} \frac{z^{n}}{n !} \int_{\left(\mathbb{R}^{d}\right)^{n}} \mathbb{I}_{\mathcal{A}}\left(\xi_{1}, \ldots, \xi_{n}\right) \mathrm{e}^{-\beta_{n}^{l, \eta}\left(\xi_{1}, \ldots, \xi_{n}\right)}\left(1-\prod_{i=1}^{n} \mathbb{I}_{\Lambda-B\left(\eta_{\left.\Lambda^{c}, r\right)}\left(\xi_{i}\right)\right) \mathrm{d} \xi_{1} \cdots \mathrm{d} \xi_{n}}\right. \\
& \leqslant \frac{\mathrm{e}^{-z 2^{d} l^{d}}}{Z_{z}^{l, \eta}} \sum_{n=0}^{+\infty} \frac{z^{n}}{n !} \int_{\left(\mathbb{R}^{d}\right)^{n}} \mathbb{I}_{\mathcal{A}}\left(\xi_{1}, \ldots, \xi_{n}\right) \mathrm{e}^{-\beta_{n}^{l, \eta}\left(\xi_{1}, \ldots, \xi_{n}\right)}\left(\sum_{i=1}^{n} \mathbb{I}_{\psi^{l, \eta}\left(\xi_{i}\right)>0}\right) \mathrm{d} \xi_{1} \cdots \mathrm{d} \xi_{n} \\
& \leqslant \frac{\mathrm{e}^{-z 2^{d} l^{d}}}{Z_{z}^{l, \eta}} \sum_{n=0}^{+\infty} \frac{z^{n}}{n !} n \int_{\left(\mathbb{R}^{d}\right)^{n} \cap \mathcal{A}} \mathrm{e}^{-\psi^{l, \eta}\left(\xi_{1}\right)} \exp (-2 \bar{N} \underline{\varphi}) \mathrm{e}^{-\beta_{n-1}^{l, \eta}\left(\xi_{2}, \ldots, \xi_{n}\right)} \mathbb{I}_{\psi^{l, \eta}\left(\xi_{1}\right)>0} \mathrm{~d} \xi_{1} \cdots \mathrm{d} \xi_{n} \\
& \leqslant \frac{\mathrm{e}^{-z 2^{d} l^{d}}}{Z_{z}^{l, \eta}} \sum_{n=1}^{+\infty} z \frac{z^{n-1}}{(n-1) !} \nu_{n-1}^{l, \eta}\left(\left(\mathbb{R}^{d}\right)^{n-1}\right) \exp (-2 \bar{N} \underline{\varphi}) \int_{\mathbb{R}^{d}} \mathbb{I}_{\psi^{l, \eta}(y)>0} \exp \left(-\psi^{l, \eta}(y)\right) \mathrm{d} y \\
& \leqslant z \exp (-2 \bar{N} \underline{\varphi}) \int_{\mathbb{R}^{d}} \mathbb{I}_{\psi^{l, \eta}(y)>0} \exp \left(-\psi^{l, \eta}(y)\right) \mathrm{d} y .
\end{aligned}
$$

In order to prove Proposition 3.1, we need the following lemma which states that, for nice trajectories only a finite number of other particles interacts with each fixed particle. Thus dynamics $(\mathcal{E})$ reduces to an infinite number of SDE involving only a finite random number of particles up to time 1.

Lemma 3.3. Let us assume that the path $X \in \mathcal{C}([0,1])$ does not belong to $\mathcal{B}(m, \rho+m, \varepsilon)$ for some $\varepsilon \in 1 / \mathbb{N}^{*}$, some $m \in \mathbb{N}^{*}$ and some $\rho \in \mathbb{N}^{*}$. For $k$ in $\{0,1, \cdots, m-1\}$ we define $J_{k}(X)$ as the set of indices $i \in \mathbb{N}$ such that either $\left|X_{i}\left(\frac{k}{m}\right)\right| \leqslant \rho+m^{2}-k m+R+\varepsilon$ or $X_{i}\left(\frac{k}{m}\right)$ belongs to a $(R+\varepsilon)$-chain of particles which intersects $B\left(0, \rho+m^{2}-k m+R+\varepsilon\right)$. Then the following inclusions hold:

$$
\left\{i \in \mathbb{N},\left|X_{i}(0)\right| \leqslant \rho\right\} \subset J_{m-1}(X) \subset \cdots \subset J_{k+1}(X) \subset J_{k}(X) \subset \cdots \subset J_{0}(X)
$$

Particles of $J_{k}(X)$ stay around the origin in the following sense:

$$
\forall i \in J_{k}(X) \quad \forall t \in\left[\frac{k}{m}, \frac{k+1}{m}\right] \quad\left|X_{i}(t)\right| \leqslant \rho+m^{2}+m+1 .
$$

They stay also far away from the others:

$$
\forall i \in J_{k}(X) \quad \forall j \notin J_{k}(X) \quad \forall t \in\left[\frac{k}{m}, \frac{k+1}{m}\right] \quad\left|X_{i}(t)-X_{j}(t)\right|>R+\frac{\varepsilon}{2} .
$$

Proof of Lemma 3.3. The set $J_{k}(X)$ is defined as the set of indices $i \in \mathbb{N}$ such that $X_{i}\left(\frac{k}{m}\right)$ belongs to $B(0, \rho+$ $\left.m^{2}-k m+R+\varepsilon\right)$ or is connected to $B\left(0, \rho+m^{2}-k m+R+\varepsilon\right)$ by some $(R+\varepsilon)$-chain of particles of $X\left(\frac{k}{m}\right)$; thus

$$
\forall j \notin J_{k}(X) \quad\left|X_{j}\left(\frac{k}{m}\right)\right|>\rho+m^{2}-k m+R+\varepsilon
$$

and

$$
\forall i \in J_{k}(X) \quad \forall j \notin J_{k}(X) \quad\left|X_{i}\left(\frac{k}{m}\right)-X_{j}\left(\frac{k}{m}\right)\right|>R+\varepsilon .
$$

Since $X \notin \mathcal{B}(m, \rho+m, \varepsilon)$ then $X\left(\frac{k}{m}\right)$ does not include any $(R+\varepsilon)$-chain of particles with diameter greater than $m-R-\varepsilon$ :

$$
\forall i \in J_{k}(X) \quad\left|X_{i}\left(\frac{k}{m}\right)\right| \leqslant\left(\rho+m^{2}-k m+R+\varepsilon\right)+(m-R-\varepsilon)=\rho+m^{2}-(k-1) m .
$$


Again since $X \notin \mathcal{B}(m, \rho+m, \varepsilon)$, no particle of $X$ entering $B\left(0, \rho+m+2 m^{2}\right)$ moves for more than $\frac{\varepsilon}{4}$ during a time interval of length $\frac{1}{m}$ :

$$
\forall i \in J_{k}(X) \quad \forall j \notin J_{k}(X) \quad \forall t \in\left[\frac{k}{m}, \frac{k+1}{m}\right] \quad\left|X_{i}(t)-X_{j}(t)\right|>R+\frac{\varepsilon}{2}
$$

and

$$
\forall i \in J_{k}(X) \quad \forall t \in\left[\frac{k}{m}, \frac{k+1}{m}\right] \quad\left|X_{i}(t)\right| \leqslant \rho+m^{2}-(k-1) m+\frac{\varepsilon}{4} \leqslant \rho+m^{2}+m+1 .
$$

Moreover

$$
\forall j \notin J_{k}(X) \quad\left|X_{j}\left(\frac{k+1}{m}\right)\right|>\rho+m^{2}-k m+R+\frac{3}{4} \varepsilon>\rho+m^{2}-k m ;
$$

using (20) this leads to

$$
j \notin J_{k}(X) \quad \Longrightarrow \quad j \notin J_{k+1}(X)
$$

which implies the decreasing property of the sets $J_{k}(X)$.

Now, using once more the small velocity property of $X$, we see that

$$
\left|X_{i}(0)\right| \leqslant \rho \Longrightarrow\left|X_{i}(1)\right| \leqslant \rho+\frac{\varepsilon}{4} m \leqslant \rho+m^{2}-(m-1) m+R+\varepsilon \Longrightarrow i \in J_{m-1}(X)
$$

and the proof is complete.

Proof of Proposition 3.1. In this whole proof, $x \in \mathcal{A}, \omega \in \Omega_{0}^{x}$ and $\rho \in \mathbb{N}^{*}$ are fixed; we also fix a corresponding $\varepsilon \in 1 / \mathbb{N}$ as in the definition of $\Omega_{0}^{x}$ and an $l \geqslant l_{0}$ associated to $\omega, \varepsilon, \rho$ as in the definition of $\Omega_{0}^{x}$. So $m(\rho, l)$ and $a(\rho, l)$ are fixed too; for simplicity they will be denoted by $m$ and $a$.

Since $X^{l, x}(\omega, \cdot) \notin \mathcal{B}(m, a, \varepsilon)$ and $X^{l+1, x}(\omega, \cdot) \notin \mathcal{B}(m, a, \varepsilon)$ with $a=m+\rho$, the results obtained in Lemma 3.3 hold for $X^{l, x}(\omega, \cdot)$ and $X^{l+1, x}(\omega, \cdot)$. In particular, recalling (13) we have for each $k$ in $\{0,1, \cdots, m-1\}$ :

$$
\forall i \in J_{k}\left(X^{l, x}(\omega, \cdot)\right) \quad \forall t \in\left[\frac{k}{m}, \frac{k+1}{m}\right] \quad\left|X_{i}^{l, x}(t)\right| \leqslant \rho+m^{2}+m+1<l-r \Longrightarrow \psi^{l, x}\left(X_{i}^{l, x}(t)\right)=0
$$

and since

$$
\forall i \in J_{k}\left(X^{l, x}(\omega, \cdot)\right) \quad \forall j \notin J_{k}\left(X^{l, x}(\omega, \cdot)\right) \quad \forall t \in\left[\frac{k}{m}, \frac{k+1}{m}\right] \quad\left|X_{i}^{l, x}(t)-X_{j}^{l, x}(t)\right|>R+\frac{\varepsilon}{2}
$$

no interaction is possible during the time interval $\left[\frac{k}{m}, \frac{k+1}{m}\right]$ between the particles of $J_{k}\left(X^{l, x}(\omega, \cdot)\right)$ and the other particles. In this case equation $\left(\mathcal{E}_{n}^{l, \eta}\right)$ verified by $X^{l, x}(\omega)$ during the time interval $\left[\frac{k}{m}, \frac{k+1}{m}\right]$ reduces to the following equation $\left(\mathcal{E}\left(k, J_{k}, X^{l, x}\right)\right)$ for the indices $i \in J_{k}\left(X^{l, x}(\omega, \cdot)\right)$ :

$$
\begin{aligned}
X_{i}^{l, x}(\omega, t)= & X_{i}^{l, x}\left(\omega, \frac{k}{m}\right)+W_{i}(\omega, t)-W_{i}\left(\omega, \frac{k}{m}\right)-\frac{1}{2} \int_{\frac{k}{m}}^{t} \sum_{j \in J_{k}\left(X^{l, x}(\omega, \cdot)\right)} \nabla \varphi\left(X_{i}^{l, x}(\omega, s)-X_{j}^{l, x}(\omega, s)\right) \mathrm{d} s \\
& +\int_{\frac{k}{m}}^{t} \sum_{j \in J_{k}\left(X^{l, x}(\omega, \cdot)\right)}\left(X_{i}^{l, x}(\omega, s)-X_{j}^{l, x}(\omega, s)\right) \mathrm{d} L_{i j}^{l, x}(\omega, s)
\end{aligned}
$$


For the same reasons, the equation $\left(\mathrm{e}^{l+1}\right)$ verified by $X^{l+1, x}(\omega, \cdot)$ during the time interval $\left[\frac{k}{m}, \frac{k+1}{m}\right]$ reduces to the following equation $\left(\mathcal{E}\left(k, J_{k}, X^{l+1, x}\right)\right)$ for the indices $i \in J_{k}\left(X^{l+1, x}(\omega, \cdot)\right)$ :

$$
\begin{aligned}
X_{i}^{l+1, x}(\omega, t)= & X_{i}^{l+1, x}\left(\omega, \frac{k}{m}\right)+W_{i}(\omega, t)-W_{i}\left(\omega, \frac{k}{m}\right)-\frac{1}{2} \int_{\frac{k}{m}}^{t} \sum_{j \in J_{k}\left(X^{l+1, x}(\omega, \cdot)\right)} \nabla \varphi\left(X_{i}^{l+1, x}(\omega, s)-X_{i}^{l+1, x}(\omega, s)\right) \mathrm{d} s \\
& +\int_{\frac{k}{m}}^{t} \sum_{j \in J_{k}\left(X^{l+1, x}(\omega, \cdot)\right)}\left(X_{i}^{l+1, x}(\omega, s)-X_{i}^{l+1, x}(\omega, s)\right) \mathrm{d} L_{i j}^{l+1, x}(\omega, s)
\end{aligned}
$$

But since $X^{l, x}(\omega, 0)=X^{l+1, x}(\omega, 0)=x$ and $L^{l, x}(\omega, 0)=L^{l+1, x}(\omega, 0)=0$, sets $J_{0}\left(X^{l, x}(\omega, \cdot)\right)$ and $J_{0}\left(X^{l+1, x}(\omega, \cdot)\right)$ are equal and equations (21) and (22) coincide for $k=0$. The strong uniqueness of the solution then implies:

$$
\begin{aligned}
\forall t \in\left[0, \frac{1}{m}\right] \quad \forall i, j \in J_{0}\left(X^{l, x}(\omega, \cdot)\right)=J_{0}\left(X^{l+1, x}(\omega, \cdot)\right) \\
X_{i}^{l, x}(\omega, t)=X_{i}^{l+1, x}(\omega, t) \text { and } L_{i j}^{l, x}(\omega, t)=L_{i j}^{l+1, x}(\omega, t)
\end{aligned}
$$

and because $J_{1}\left(X^{l, x}(\omega, \cdot)\right) \subset J_{0}\left(X^{l, x}(\omega, \cdot)\right)$ (and idem for $J_{1}\left(X^{l+1, x}(\omega, \cdot)\right)$ ) this in turn implies that $J_{1}\left(X^{l, x}(\omega, \cdot)\right)=J_{1}\left(X^{l+1, x}(\omega, \cdot)\right)$. But again, since

$\forall i, j \in J_{1}\left(X^{l, x}(\omega, \cdot)\right)=J_{1}\left(X^{l+1, x}(\omega, \cdot)\right) \quad X_{i}^{l, x}\left(\omega, \frac{1}{m}\right)=X_{i}^{l+1, x}\left(\omega, \frac{1}{m}\right)$ and $L_{i j}^{l, x}\left(\omega, \frac{1}{m}\right)=L_{i j}^{l+1, x}\left(\omega, \frac{1}{m}\right)$

equations $(21)$ and $(22)$ coincide for $k=1$, and the strong uniqueness implies $X_{i}^{l, x}(\omega,.) \equiv X_{i}^{l+1, x}(\omega,$. (and $\left.L_{i j}^{l, x}(\omega,.) \equiv L_{i j}^{l+1, x}(\omega,).\right)$ on $\left[\frac{1}{m}, \frac{2}{m}\right]$ for $i$ in $J_{1}\left(X^{l, x}(\omega, \cdot)\right)=J_{1}\left(X^{l+1, x}(\omega, \cdot)\right.$, which in turn implies that $J_{2}\left(X^{l, x}(\omega, \cdot)\right)=J_{2}\left(X^{l+1, x}(\omega, \cdot)\right.$.

By induction, we thus obtain that for all $k \in\{1, \ldots, m-1\}, J_{k}\left(X^{l, x}(\omega, \cdot)\right)=J_{k}\left(X^{l+1, x}(\omega, \cdot)\right)$ and

$$
\forall i, j \in J_{k}\left(X^{l, x}(\omega, \cdot)\right), t \in\left[0, \frac{k+1}{m}\right], X_{i}^{l, x}(\omega, t)=X_{i}^{l+1, x}(\omega, t) \text { and } L_{i j}^{l, x}(\omega, t)=L_{i j}^{l+1, x}(\omega, t) .
$$

Using the inclusion chain $\left\{i \in \mathbb{N},\left|x_{i}\right| \leqslant \rho\right\} \subset J_{m-1}(X) \subset \cdots \subset J_{1}(X) \subset J_{0}(X)$ which holds for $X=X^{l, x}(\omega, \cdot)$ and $X=X^{l+1, x}(\omega, \cdot)$ because $X^{l, x}(\omega, 0)=X^{l+1, x}(\omega, 0)=x$, we obtain that $X_{i}^{l, x}(\omega, \cdot)$ and $X_{i}^{l+1, x}(\omega, \cdot)$ are equal on $[0,1]$ for $i$ 's such that $\left|x_{i}\right| \leqslant \rho$ and the same result holds for $\left(L_{i j}^{l, x}(\omega, \cdot)\right)_{i, j}$ and $\left(L_{i j}^{l+1, x}(\omega, \cdot)\right)_{i, j}$ because both local times coincide if $j$ in $J_{0}\left(X^{l, x}(\omega, \cdot)\right)$ and identically vanish otherwise.

Since $\rho$ may be chosen arbitrary large, Proposition 3.1 is proven.

\section{Remaining PROOFS}

The main Theorems 1.3, 1.4 and 1.5 are now direct consequences of Propositions 4.1, 4.4 and 4.5 enounced and proved in this section.

In order to prove these propositions, we need some more notations. We first fix $x \in \mathcal{A}$. For $\tilde{m} \in \mathbb{N}^{*}, \tilde{a} \geqslant 1$ and $\varepsilon \in 1 / \mathbb{N}$ fixed, let $\Omega^{x}(\tilde{m}, \tilde{a}, \varepsilon)$ be the set of $\omega^{\prime}$ s such that $X^{l, x}(\omega, \cdot)$ does not belong to $\tilde{\mathcal{B}}(\tilde{m}, \tilde{a}, \varepsilon)$ for an infinite number of indices $l$ :

$$
\Omega^{x}(\tilde{m}, \tilde{a}, \varepsilon)=\left\{\omega \in \Omega: \forall p \in \mathbb{N} \exists l \geqslant p, \quad X^{l, x}(\omega, .) \notin \tilde{\mathcal{B}}(\tilde{m}, \tilde{a}, \varepsilon)\right\}=\limsup _{l \rightarrow+\infty}\left\{X^{l, x} \notin \tilde{\mathcal{B}}(\tilde{m}, \tilde{a}, \varepsilon)\right\}
$$


We also define

$$
\begin{aligned}
\Omega_{1}^{x} & =\left\{\omega \in \Omega \text { s.t. } \forall \varepsilon \in 1 / \mathbb{N} \text { for } \rho \text { large enough and for an infinite number of l's } X^{l, x} \notin \tilde{\mathcal{B}}(\rho, R, \varepsilon)\right\} \\
& =\bigcap_{\varepsilon \in 1 / \mathbb{N}} \liminf _{\rho \rightarrow+\infty} \Omega^{x}(\rho, R, \varepsilon) .
\end{aligned}
$$

We have the following result:

Proposition 4.1. For every $x \in \mathcal{A}$ and $\omega \in \Omega_{0}^{x} \cap \Omega_{1}^{x}$, the process $\left(X^{\infty, x}(\omega,),. L_{i j}^{\infty, x}(\omega,).\right)$ satisfies equation $(\mathcal{E})$ with $X^{\infty, x}(\omega, 0)=x$. Thus, for any $x \in \underline{\underline{\mathcal{A}}}=\left\{\xi \in \mathcal{A}: P\left(\Omega_{0}^{\xi} \cap \Omega_{1}^{\xi}\right)=1\right\}$, the process $\left(X^{\infty, x}, L_{i j}^{\infty, x}\right)$ is a solution of $(\mathcal{E})$ with initial condition $x$. Moreover for each $z<z_{c}$ and $\mu \in \mathcal{G}(z) \quad \mu(\underline{\underline{A}})=1$.

Before proving this proposition, we first establish some useful results on $\Omega^{x}(\tilde{m}, \tilde{a}, \varepsilon)$ and $\Omega_{1}^{x}$.

Lemma 4.2. Let $\mu$ be a Gibbsian measure in $\mathcal{G}(z)$. For each $\varepsilon \in 1 / \mathbb{N}, \tilde{m} \in \mathbb{N}^{*}$ and $\tilde{a} \geqslant 1$, one has

$$
\int P\left(\Omega^{x}(\tilde{m}, \tilde{a}, \varepsilon)^{c}\right) \mathrm{d} \mu(x) \leqslant z C_{d}^{\prime} \mathrm{e}^{-2 \bar{N} \underline{\varphi}} \tilde{a}^{d} \tilde{m}^{2 d} \exp \left(-\frac{\varepsilon^{2}}{96} \tilde{m}\right) .
$$

As corollary

$$
\int P\left(\Omega_{1}^{x}\right) \mathrm{d} \mu(x)=1
$$

Lemma 4.3. For each $\tilde{m} \in \mathbb{N}^{*}, \tilde{a} \geqslant 1$ and $\varepsilon \in 1 / \mathbb{N}$ one also has

$$
\forall \omega \in \Omega^{x}(\tilde{m}, \tilde{a}, \varepsilon) \quad X^{\infty, x}(\omega, \cdot) \notin \tilde{\mathcal{B}}(\tilde{m}, \tilde{a}, \varepsilon)
$$

and consequently

$$
\forall x \in \mathcal{A} \quad \forall \omega \in \Omega_{1}^{x} \quad \forall \varepsilon \in 1 / \mathbb{N} \quad \exists \rho_{0} \text { s.t. } \forall \rho \geqslant \rho_{0} \quad X^{\infty, x}(\omega, \cdot) \notin \tilde{\mathcal{B}}(\rho, R, \varepsilon) .
$$

Proof of Lemma 4.2. By definition of $\Omega(\tilde{m}, \tilde{a}, \varepsilon)$ one has

$$
\Omega^{x}(\tilde{m}, \tilde{a}, \varepsilon)^{c}=\liminf _{l \rightarrow+\infty}\left\{\omega \in \Omega \text { s.t. } X^{l, x}(\omega, \cdot) \in \tilde{\mathcal{B}}(\tilde{m}, \tilde{a}, \varepsilon)\right\} .
$$

By Fatou lemma

$$
\int P\left(\Omega^{x}(\tilde{m}, \tilde{a}, \varepsilon)^{c}\right) \mathrm{d} \mu(x) \leqslant \liminf _{l \rightarrow+\infty} \int P\left(X^{l, x}(\omega, \cdot) \in \tilde{\mathcal{B}}(\tilde{m}, \tilde{a}, \varepsilon)\right) \mathrm{d} \mu(x) .
$$

Using inequality (17) (see the proof of Prop. 3.2 step 1) applied to the event $\Theta=\tilde{\mathcal{B}}(\tilde{m}, \tilde{a}, \varepsilon)$ we obtain the following bound:

$$
\int P\left(\Omega^{x}(\tilde{m}, \tilde{a}, \varepsilon)^{c}\right) \mathrm{d} \mu(x) \leqslant \liminf _{l \rightarrow+\infty} \int_{\mathcal{A}} Q_{z}^{l, \eta}(\tilde{\mathcal{B}}(\tilde{m}, \tilde{a}, \varepsilon)) \mathrm{d} \mu(\eta)+2 \int_{\mathcal{A}}\left(1-\frac{Z_{z}^{\Lambda, \eta}}{Z_{z}^{l, \eta}}\right) \mathrm{d} \mu(\eta) ;
$$

thus by Proposition 2.6

$$
\begin{aligned}
& \int P\left(\Omega^{x}(\tilde{m}, \tilde{a}, \varepsilon)^{c}\right) \mathrm{d} \mu(x) \\
& \leqslant \int_{\mathcal{A}} z C_{d}^{\prime} \mathrm{e}^{-2 \bar{N} \underline{\varphi}} \tilde{a}^{d} \tilde{m}^{2 d} \exp \left(-\frac{\varepsilon^{2}}{96} \tilde{m}\right) \mathrm{d} \mu(\eta)+2 \lim _{l \rightarrow+\infty} \int_{\mathcal{A}}\left(1-\frac{Z_{z}^{\Lambda, \eta}}{Z_{z}^{l, \eta}}\right) \mathrm{d} \mu(\eta) .
\end{aligned}
$$


Inequality (18) implies that $\lim _{l \rightarrow+\infty} \int_{\mathcal{A}}\left(1-\frac{Z_{z}^{\Lambda, \eta}}{Z_{z}^{l, \eta}}\right) \mathrm{d} \mu(\eta)=0$ thus

$$
\int P\left(\Omega^{x}(\tilde{m}, \tilde{a}, \varepsilon)^{c}\right) \mathrm{d} \mu(x) \leqslant z C_{d}^{\prime} \mathrm{e}^{-2 \bar{N} \underline{\varphi}} \tilde{a}^{d} \tilde{m}^{2 d} \exp \left(-\frac{\varepsilon^{2}}{96} \tilde{m}\right)
$$

Replacing $\tilde{m}$ and $\tilde{a}$ by $\rho$ and $R$ in the above inequality, we obtain:

$$
\forall \varepsilon \in 1 / \mathbb{N} \quad \sum_{\rho=1}^{+\infty} \int P\left(\Omega^{x}(\rho, R, \varepsilon)^{c}\right) \mathrm{d} \mu(x)<+\infty .
$$

By Borel-Cantelli lemma, this leads to:

$$
\forall \varepsilon \in 1 / \mathbb{N} \quad \int P\left(\limsup _{\rho \rightarrow+\infty} \Omega^{x}(\rho, R, \varepsilon)^{c}\right) \mathrm{d} \mu(x)=0
$$

and consequently

$$
\int P\left(\left(\Omega_{1}^{x}\right)^{c}\right) \mathrm{d} \mu(x)=\int P\left(\bigcup_{\varepsilon \in 1 / \mathbb{N}} \limsup _{\rho \rightarrow+\infty} \Omega^{x}(\rho, R, \varepsilon)^{c}\right) \mathrm{d} \mu(x)=0 .
$$

Proof of Lemma 4.3. According to Proposition 3.1

$$
\forall x \in \mathcal{A} \quad \forall \omega \in \Omega_{0}^{x} \quad \forall i \in \mathbb{N} \quad \exists l_{0} \in \mathbb{N} \quad \forall l \geqslant l_{0} \quad X_{i}^{\infty, x}(\omega, \cdot) \equiv X_{i}^{l, x}(\omega, \cdot) \text { on }[0,1]
$$

Consequently, for $x \in \mathcal{A}$ and $\omega \in \Omega^{x}(\tilde{m}, \tilde{a}, \varepsilon) \cap \Omega_{0}^{x}$ and for $i \in \mathbb{N}$, there exists an $l \geqslant l_{0}$ such that $X^{l, x}(\omega, \cdot) \notin$ $\tilde{\mathcal{B}}(\tilde{m}, \tilde{a}, \varepsilon)$, i.e.

$$
X_{i}^{\infty, x}(\omega, \cdot)=X_{i}^{l, x}(\omega, \cdot) \text { on }[0,1] \quad \text { and } \quad w\left(X_{i}^{l, x}(\omega, \cdot), \frac{1}{\tilde{m}}\right) \leqslant \frac{\varepsilon}{4} \text { or } \forall t \leqslant 1,\left|X_{i}^{l, x}(\omega, t)\right|>\tilde{a}+2 \tilde{m}^{2}
$$

Thus

$$
\forall x \in \mathcal{A} \quad \forall \omega \in \Omega^{x}(\tilde{m}, \tilde{a}, \varepsilon) \cap \Omega_{0}^{x} \quad \forall i \in \mathbb{N} \quad w\left(X_{i}^{\infty, x}(\omega, \cdot), \frac{1}{\tilde{m}}\right) \leqslant \frac{\varepsilon}{4} \text { or } \forall t \leqslant 1,\left|X_{i}^{\infty, x}(\omega, t)\right|>\tilde{a}+2 \tilde{m}^{2}
$$

By definition of $\Omega_{1}^{x}$,

$$
\forall x \in \mathcal{A} \quad \forall \omega \in \Omega_{0}^{x} \cap \Omega_{1}^{x} \quad \forall \varepsilon \in 1 / \mathbb{N} \quad \exists \rho_{0} \text { s.t. } \forall \rho \geqslant \rho_{0} \quad \omega \in \Omega^{x}(\rho, R, \varepsilon) .
$$

So for any $x \in \mathcal{A}, \omega \in \Omega_{0}^{x} \cap \Omega_{1}^{x}$ and $\varepsilon \in 1 / \mathbb{N}$ there exists $\rho_{0}$ such that

$$
\forall \rho \geqslant \rho_{0} \quad \forall i \in \mathbb{N} w\left(X_{i}^{\infty, x}(\omega, \cdot), \frac{1}{\rho}\right) \leqslant \frac{\varepsilon}{4} \text { or } \forall t \leqslant 1,\left|X_{i}^{\infty, x}(\omega, t)\right|>R+2 \rho^{2}
$$

that is

$$
\forall x \in \mathcal{A} \quad \forall \omega \in \Omega_{0}^{x} \cap \Omega_{1}^{x} \quad \forall \varepsilon \in 1 / \mathbb{N} \quad \exists \rho_{0} \text { s.t. } \forall \rho \geqslant \rho_{0} \quad X^{\infty, x}(\omega, \cdot) \notin \tilde{\mathcal{B}}(\rho, R, \varepsilon) .
$$


Proof of Proposition 4.1. Let us fix $\mu \in \mathcal{G}(z)$ for some $z<z_{c}$. As corollary of Proposition 3.2 and Lemma 4.2 ,

$$
\int P\left(\Omega_{0}^{x} \cap \Omega_{1}^{x}\right) \mathrm{d} \mu(x)=1 .
$$

This proves that for $\mu$-almost every $x$ in $\mathcal{A}, P\left(\Omega_{0}^{x} \cap \Omega_{1}^{x}\right)=1$ and then $\mu(\underline{\mathcal{A}})=1$.

We fix an $x \in \mathcal{A}$ such that $P\left(\Omega_{0}^{x} \cap \Omega_{1}^{x}\right)=1$ and $\omega \in \Omega_{0}^{x} \cap \Omega_{1}^{x}$.

We first use the fact that $\omega \in \Omega_{0}^{x}$. For $\varepsilon \in 1 / \mathbb{N}$ smaller than $\varepsilon_{0}$ corresponding to $\omega$ in the definition of $\Omega_{0}^{x}$, for each $\rho \in \mathbb{N}^{*}$ and $l \geqslant \rho+1$ greater than $l_{0}$ associated to $\omega, \varepsilon, \rho$, we have $X^{l, x}(\omega, \cdot) \notin \mathcal{B}(m(\rho, l), \rho+m(\rho, l), \varepsilon)$. Lemma 3.3 and inequality (13) then imply, as in the proof of Proposition 3.1(i), that $\left|X_{i}^{l, x}(\omega, t)\right|<l-r$ for $t \in[0,1]$ and for $i$ 's such that $\left|x_{i}\right| \leqslant \rho$. Equation $\left(\mathcal{E}_{n}^{l, \eta}\right)$ then reduces to the simpler equation:

$$
\begin{gathered}
\forall \omega \in \Omega_{0}^{x} \cap \Omega_{1}^{x} \quad \forall \rho \in \mathbb{N}^{*} \quad \exists l_{0} \text { s.t. } \forall l \geqslant l_{0} \quad \forall i \text { s.t. }\left|x_{i}\right| \leqslant \rho \quad \forall t \in[0,1] \\
\begin{aligned}
X_{i}^{l, x}(\omega, t)= & x_{i}+W_{i}(\omega, t)-\frac{1}{2} \int_{0}^{t} \sum_{j \in \mathbb{N}} \nabla \varphi\left(X_{i}^{l, x}(\omega, s)-X_{j}^{l, x}(\omega, s)\right) \mathrm{d} s \\
& +\int_{0}^{t} \sum_{j \in \mathbb{N}}\left(X_{i}^{l, x}(\omega, s)-X_{j}^{l, x}(\omega, s)\right) \mathrm{d} L_{i j}^{l, x}(\omega, s) .
\end{aligned}
\end{gathered}
$$

Since $\omega$ belongs to $\Omega_{1}^{x}$ too there exists $\varepsilon \in 1 / \mathbb{N}^{*}$ and $\rho_{0}$ such that $\omega \in \Omega^{x}(\rho, R, \varepsilon)$ for each $\rho \geqslant \rho_{0}$. Let us fix such a $\rho$. Since $\omega \in \Omega^{x}(\rho, R, \varepsilon)$, there exists an infinite number of indices $l$ such that $X^{l, x}(\omega, \cdot) \notin \tilde{\mathcal{B}}(\rho, R, \varepsilon)$. Remark that $R+2 \rho^{2} \geqslant \rho+\frac{\varepsilon}{4} \rho+R$ so for $l$ 's such that $X^{l, x}(\omega, \cdot) \notin \tilde{\mathcal{B}}(\rho, R, \varepsilon)$ we have:

$$
\begin{aligned}
& \forall i \in \mathbb{N} \quad\left|x_{i}\right| \leqslant \rho \quad \Longrightarrow \quad \forall t \in[0,1] \quad\left|X_{i}^{l, x}(\omega, t)\right| \leqslant \rho+\frac{\varepsilon}{4} \rho \\
& \forall j \in \mathbb{N} \quad\left|x_{i}\right|>\rho+\frac{\varepsilon}{2} \rho+R \quad \Longrightarrow \quad \forall t \in[0,1] \quad\left|X_{j}^{l, x}(\omega, t)\right|>\rho+\frac{\varepsilon}{4} \rho+R .
\end{aligned}
$$

Equation (24) holds for these indices $l$ provided $l \geqslant l_{0}(\omega, \rho, \varepsilon)$ and $l \geqslant \rho+1$ and in this case we may replace the sums over $j \in \mathbb{N}$ by sums over $\left\{j,\left|x_{j}\right| \leqslant \rho+\frac{\varepsilon}{2} \rho+R\right\}$, due to $(25)$ :

$$
\begin{aligned}
& \forall \omega \in \Omega_{0}^{x} \cap \Omega_{1}^{x} \quad \forall \varepsilon \in 1 / \mathbb{N} \quad \exists \rho_{0} \text { s.t. } \forall \rho \geqslant \rho_{0} \\
& \text { for an infinite number of } l \text { 's and for all } i \text { s.t. }\left|x_{i}\right| \leqslant \rho \quad \forall t \in[0,1] \\
& X_{i}^{l, x}(\omega, t)=x_{i}+W_{i}(\omega, t)-\frac{1}{2} \int_{0}^{t} \sum_{\left\{j:\left|x_{j}\right| \leqslant \rho+\frac{\varepsilon}{2} \rho+R\right\}} \nabla \varphi\left(X_{i}^{l, x}(\omega, s)-X_{j}^{l, x}(\omega, s)\right) \mathrm{d} s \\
& \quad+\int_{0}^{t} \sum_{\left\{j:\left|x_{j}\right| \leqslant \rho+\frac{\varepsilon}{2} \rho+R\right\}}\left(X_{i}^{l, x}(\omega, s)-X_{j}^{l, x}(\omega, s)\right) \mathrm{d} L_{i j}^{l, x}(\omega, s) .
\end{aligned}
$$

Since the set $\left\{j:\left|x_{j}\right| \leqslant \rho+\frac{\varepsilon}{2} \rho+R\right\}$ is finite, using proposition 3.1 we can choose $l$ large enough such that (26) holds and

Consequently

$$
\forall j \text { such that }\left|x_{j}\right| \leqslant \rho+\frac{\varepsilon}{2} \rho+R \quad X_{j}^{l, x}(\omega, \cdot)=X_{j}^{\infty, x}(\omega, \cdot) \text { on }[0,1] \text {. }
$$

$$
\begin{aligned}
& \forall \omega \in \Omega_{0}^{x} \cap \Omega_{1}^{x} \quad \forall \varepsilon \in 1 / \mathbb{N} \quad \exists \rho_{0} \text { s.t. } \forall \rho \geqslant \rho_{0} \quad \forall i \text { s.t. }\left|x_{i}\right| \leqslant \rho \quad \forall t \in[0,1] \\
& X_{i}^{\infty, x}(\omega, t)=x_{i}+W_{i}(\omega, t)-\frac{1}{2} \int_{0}^{t} \sum_{j,\left|x_{j}\right| \leqslant \rho+\frac{\varepsilon}{2} \rho+R} \nabla \varphi\left(X_{i}^{\infty, x}(\omega, s)-X_{j}^{\infty, x}(\omega, s)\right) \mathrm{d} s \\
& \quad+\int_{0}^{t} \sum_{j,\left|x_{j}\right| \leqslant \rho+\frac{\varepsilon}{2} \rho+R}\left(X_{i}^{\infty, x}(\omega, s)-X_{j}^{\infty, x}(\omega, s)\right) \mathrm{d} L_{i j}^{\infty, x}(\omega, s) .
\end{aligned}
$$


On the other hand, since $\omega \in \Omega^{x}(\rho, R, \varepsilon)$ for each $\rho \geqslant \rho_{0}$, Lemma 4.3 leads to $X^{\infty, x}(\omega, \cdot) \notin \tilde{\mathcal{B}}(\rho, R, \varepsilon)$. As already remarked for $X^{l, x}(\omega, \cdot)$, this implies that it is equivalent to sum over $j \in \mathbb{N}$ or over $\left\{j,\left|x_{j}\right| \leqslant \rho+\frac{\varepsilon}{2} \rho+R\right\}$ in the above equation:

$$
\begin{gathered}
\forall \omega \in \Omega_{0}^{x} \cap \Omega_{1}^{x} \quad \forall \varepsilon \in 1 / \mathbb{N} \quad \exists \rho_{0} \text { s.t. } \forall \rho \geqslant \rho_{0} \quad \forall i \text { s.t. }\left|x_{i}\right| \leqslant \rho \quad \forall t \in[0,1] \\
\begin{aligned}
X_{i}^{\infty, x}(\omega, t)= & x_{i}+W_{i}(\omega, t)-\frac{1}{2} \int_{0}^{t} \sum_{j \in \mathbb{N}} \nabla \varphi\left(X_{i}^{\infty, x}(\omega, s)-X_{j}^{\infty, x}(\omega, s)\right) \mathrm{d} s \\
& +\int_{0}^{t} \sum_{j \in \mathbb{N}}\left(X_{i}^{\infty, x}(\omega, s)-X_{j}^{\infty, x}(\omega, s)\right) \mathrm{d} L_{i j}^{\infty, x}(\omega, s)
\end{aligned}
\end{gathered}
$$

i.e. for all $\omega \in \Omega_{0}^{x} \cap \Omega_{1}^{x}, i \in \mathbb{N}$ and $t \in[0,1]$

$$
\begin{aligned}
X_{i}^{\infty, x}(\omega, t) & =x_{i}+W_{i}(\omega, t)-\frac{1}{2} \int_{0}^{t} \sum_{j \in \mathbb{N}} \nabla \varphi\left(X_{i}^{\infty, x}(\omega, s)-X_{j}^{\infty, x}(\omega, s)\right) \mathrm{d} s \\
& +\int_{0}^{t} \sum_{j \in \mathbb{N}}\left(X_{i}^{\infty, x}(\omega, s)-X_{j}^{\infty, x}(\omega, s)\right) \mathrm{d} L_{i j}^{\infty, x}(\omega, s) .
\end{aligned}
$$

This prove that $X^{\infty, x}$ is a solution of $(\mathcal{E})$ starting from $x$.

Proposition 4.4. The process $\left(X_{i}^{\infty, x}(t), L_{i j}^{\infty, x}(t), i, j \in \mathbb{N}, t \in \mathbb{R}^{+}\right)$is the unique solution of equation $(\mathcal{E})$ with initial point $x \in \underline{\mathcal{A}}$ inside the class of paths $\mathcal{C}$ defined as follows:

$X \in \mathcal{C}\left(\mathbb{R}^{+}, \mathcal{A}\right)$ belongs to $\mathcal{C}$ if there exists $\varepsilon>0$ and $p \in \mathbb{N}^{*}$ such that for all $\rho, m_{0} \in \mathbb{N}^{*}$ there exists an integer $m \geqslant m_{0}$, a sequence $0=t_{0}<t_{1}<\cdots<t_{m^{\prime}}=1$ in $\mathbb{Q}$ verifying $t_{k+1}-t_{k} \leqslant \frac{1}{m}$ and bounded open sets $C_{0}, C_{1}, \cdots, C_{m^{\prime}-1}$ in $\mathbb{R}^{d}$ which satisfy

$$
\begin{aligned}
& B(0, \rho+m) \subset C_{m^{\prime}-1} \subset B\left(C_{m^{\prime}-1}, \varepsilon\right) \subset C_{m^{\prime}-2} \subset \cdots \subset B\left(C_{1}, \varepsilon\right) \subset C_{0} \subset B\left(0, \rho+m+m^{p}\right) \\
& \text { and } \forall k \in\left\{0, \cdots, m^{\prime}-1\right\} \quad d\left(\left\{X_{j}(u), j \in \mathbb{N}^{*}, u \in\left[t_{k}, t_{k+1}\right]\right\}, \partial C_{k}\right) \geqslant \frac{R}{2}+\frac{\varepsilon}{4} .
\end{aligned}
$$

Proof. We first check that for $\omega \in \Omega_{0}^{x}, X^{\infty, x}(\omega, \cdot) \in \mathcal{C}$. To this aim, we choose $\varepsilon=\varepsilon_{0} \leqslant R$ as in the definition of $\Omega_{0}^{x}$ and $p=2$. For each $\rho$ and $m_{0}$ in $\mathbb{N}^{*}$, one may find $l \geqslant l_{0}(\omega, \rho, \varepsilon)$ large enough to have $m(\rho, l) \geqslant m_{0}$. Then $m=m(\rho, l), m^{\prime}=m, t_{k}=\frac{k}{m}$ and

$$
C_{k}=B\left(0, \rho+m 2-k m+\frac{R+\varepsilon}{2}\right) \cup \bigcup_{i \in J_{k}\left(X^{\infty, x}(\omega, \cdot)\right)} B\left(X_{i}^{\infty, x}\left(\omega, \frac{k}{m}\right), \frac{R+\varepsilon}{2}\right)
$$

are convenient choices: recall that Lemma 3.3 and the proof of Proposition 3.1 imply that

$$
d\left(\left\{X_{j}^{\infty, x}(u), j \in \mathbb{N}^{*}, u \in\left[\frac{k}{m}, \frac{k+1}{m}\right]\right\}, \partial C_{k}\right) \geqslant \frac{R}{2}+\frac{\varepsilon_{0}}{4}
$$

and that $B\left(C_{k}, \varepsilon\right) \subset B\left(0, \rho+m 2-(k-1)+m+\frac{\varepsilon}{4}+\varepsilon\right) \subset C_{k-1}$.

The proof of uniqueness is then a direct generalization of the proof of uniqueness for hard core potential made by Tanemura [8], Lemma 5.4; so we omit it.

Proposition 4.5. If the initial configuration of $X^{\infty, x}$ is random with distribution $\mu \in \mathcal{G}(z)$, then this solution is time-reversible, that is its law is invariant with respect to the time reversal. 
Proof. We have to prove that for any $T \in[0,1]$, for $f_{1}, \ldots, f_{k}$ bounded continuous functions on $\mathcal{M}$ with compact support and for $t_{1}, \ldots, t_{k} \in[0, T]$

$$
\int E\left(\prod_{i=1}^{k} f_{i}\left(X^{\infty, x}\left(t_{i}\right)\right)\right) \mathrm{d} \mu(x)=\int E\left(\prod_{i=1}^{k} f_{i}\left(X^{\infty, x}\left(T-t_{i}\right)\right)\right) \mathrm{d} \mu(x)
$$

But $X^{\infty, x}$ is, by construction, the weak limit of $X^{l, x}$. Then equality (28) holds if the following equality holds:

$$
\lim _{l \rightarrow+\infty} \int E\left(\prod_{i=1}^{k} f_{i}\left(X^{l, x}\left(t_{i}\right)\right)-\prod_{i=1}^{k} f_{i}\left(X^{l, x}\left(T-t_{i}\right)\right)\right) \mathrm{d} \mu(x)=0
$$

Like in the proof of Proposition 3.2 step 1 (cf inequalities (17) and (18)), we go back to $Q_{z}^{l, \eta}$, which is reversible:

$$
\begin{aligned}
& \left|\int E\left(\prod_{i=1}^{k} f_{i}\left(X^{l, x}\left(t_{i}\right)\right)-\prod_{i=1}^{k} f_{i}\left(X^{l, x}\left(T-t_{i}\right)\right)\right) \mathrm{d} \mu(x)\right| \\
& \leqslant\left|\int_{\mathcal{A}} \int_{\mathcal{A}} \prod_{i=1}^{k} f_{i}\left(X\left(t_{i}\right)\right)-\prod_{i=1}^{k} f_{i}\left(X\left(T-t_{i}\right)\right) \mathrm{d} Q_{z}^{l, \eta}(X) \mathrm{d} \mu(\eta)\right| \\
& \quad+2 \prod_{i=1}^{k} \sup _{\xi \in \mathcal{A}}\left|f_{i}(\xi)\right| \int_{\mathcal{A}}\left(1-\frac{Z_{z}^{\Lambda, \eta}}{Z_{z}^{l, \eta}}\right) \mathrm{d} \mu(\eta)
\end{aligned}
$$

where $\Lambda=[-l, l]^{d}$. The first term of the right hand side is equal to 0 and the second term tends to zero as $l$ tends to infinity.

Acknowledgements. For the completion of this work the authors benefited partly from the financial support of the German Academic Exchange Service DAAD and the French Foreign Ministry (Procope agreement Nr. D/0333682) and also from the scientific programme "Phase Transitions and Fluctuation Phenomena for Random Dynamics in Spatially Extended Systems" from the European Science Foundation. All these institutions are here gratefully acknowledged.

\section{Appendix: estimate of the probability of FAst oscillation FOR BROWNIAN MOTION}

Proposition 5.1. If $W$ is a one-dimensional Brownian motion on $(\Omega, \mathcal{F}, P)$ then for every $\varepsilon>0$ and every $\delta \in] 0,1]$

$$
P(w(W, \delta) \geqslant \varepsilon) \leqslant \frac{41}{\delta} \exp \left(-\frac{\varepsilon^{2}}{5 \delta}\right)
$$

Proof. We first use Doob's inequality applied to the submartingale $\exp \left(2 W(\cdot)^{2} / 5 s_{0}\right)$ and then the Gaussian property $E\left(\exp \left(a W(1)^{2}\right)\right)=1 / \sqrt{1-2 a}$ to obtain

$$
\begin{aligned}
P\left(\exists s \leqslant s_{0},|W(s)| \geqslant \beta\right) & =P\left(\sup _{0 \leqslant s \leqslant s_{0}} \exp \left(\frac{2 W(s)^{2}}{5 s_{0}}\right) \geqslant \exp \left(\frac{2 \beta^{2}}{5 s_{0}}\right)\right) \\
& \leqslant \exp \left(-\frac{2 \beta^{2}}{5 s_{0}}\right) E\left(\exp \left(\frac{2 W\left(s_{0}\right)^{2}}{5 s_{0}}\right)\right)=\sqrt{5} \exp \left(-\frac{2 \beta^{2}}{5 s_{0}}\right) .
\end{aligned}
$$


Now using the translation invariance property of the Brownian motion and inequality (29) one obtains:

$$
\begin{aligned}
& P(w(W, \delta) \geqslant \varepsilon)=P\left(\sup _{|t-s|<\delta}|W(t)-W(s)| \geqslant \varepsilon\right) \\
& \leqslant P\left(\exists i \in \{ 0 , \frac { \delta } { 8 } , \frac { 2 \delta } { 8 } , \frac { 3 \delta } { 8 } , \cdots \} \cap [ 0 , 1 ] \exists s \in \left[i, i+\frac{\delta}{8}[\exists t \in[s, s+\delta[\text { s.t. }|W(t)-W(s)| \geqslant \varepsilon)\right.\right. \\
& \leqslant\left(\left[\frac{8}{\delta}\right]+1\right) P\left(\exists s \in \left[0, \frac{\delta}{8}\left[\exists t \in \left[0, \frac{\delta}{8}+\delta[\text { s.t. }|W(t)-W(s)| \geqslant \varepsilon)\right.\right.\right.\right. \\
& \leqslant\left(\frac{8}{\delta}+1\right) P\left(\exists s \in \left[0, \frac{\delta}{8}\left[\exists t \in \left[0, \frac{9 \delta}{8}\left[\text { s.t. }|W(s)| \geqslant \frac{\varepsilon}{4} \text { or }|W(t)| \geqslant \frac{3 \varepsilon}{4}\right)\right.\right.\right.\right. \\
& \leqslant\left(\frac{8}{\delta}+1\right)\left(\sqrt{5} \exp \left(-\frac{2}{5} \frac{\varepsilon^{2}}{16} \frac{8}{\delta}\right)+\sqrt{5} \exp \left(-\frac{2}{5} \frac{9 \varepsilon^{2}}{16} \frac{8}{9 \delta}\right)\right) \\
& \leqslant \frac{41}{\delta} \exp \left(-\frac{\varepsilon^{2}}{5 \delta}\right) .
\end{aligned}
$$

\section{REFERENCES}

[1] R.L. Dobrushin, Gibbsian random fields. The general case. Functional Anal. Appl. 3 (1969) 22-28.

[2] M. Fradon and S. Rœelly, Infinite dimensional diffusion processes with singular interaction. Bull. Sci. math. 124 (2000) $287-318$.

[3] J. Fritz, Gradient Dynamics of Infinite Points Systems. Ann Probab. 15 (1987) 478-514.

[4] H.-O. Georgii, Canonical Gibbs measures. Lecture Notes in Mathematics 760, Springer-Verlag, Berlin (1979).

[5] R. Lang, Unendlich-dimensionale Wienerprozesse mit Wechselwirkung. Z. Wahrsch. Verw. Geb. 38 (1977) 55-72.

[6] D. Ruelle, Superstable Interactions in Classical Statistical Mechanics. Comm. Math. Phys. 18 (1970) 127-159.

[7] Y. Saisho and H. Tanaka, Stochastic Differential Equations for Mutually Reflecting Brownian Balls. Osaka J. Math. 23 (1986) $725-740$.

[8] H. Tanemura, A System of Infinitely Many Mutually Reflecting Brownian Balls. Probability Theory and Related Fields 104 (1996) 399-426. 